\title{
SHRUBS IN THE MANDELBROT SET ORDERING*
}

\author{
M. Romera, G. Pastor, G. Álvarez and F. Montoya \\ Instituto de Física Aplicada, \\ Consejo Superior de Investigaciones Científicas \\ Serrano144, 28006 Madrid, Spain
}

December 16, 2004

\begin{abstract}
We introduce shrubs in this paper in order to present a first approach to studying the structure of the Mandelbrot set. Primary, secondary ... and $N$-ary shrubs are analyzed. We have experimentally obtained formulae to calculate the periods of the hyperbolic components representatives of the structural branches, and the preperiods and periods of both the nodes -where structural branches emanate- and the tips -where the shrub enters in crisis. A generalization allows us to give in each case one formula to calculate representative periods and node and tips preperiods and periods.
\end{abstract}

\section{Introduction}

Nonlinear dynamical systems have been broadly studied by physicists on account of their wide range of applications in a large number of nonlinear problems. Thus, the first physics orientated study of chaotic dynamics was presented by Lorenz [1963], and Ruelle and Takens [1971] suggested that turbulent flow might be an example of dynamical chaos. In following years, dynamical systems were applied to pattern formation in natural systems [Crutchfield and Kaneco, 1987]. Nowadays, the interest in this topic continues as we can see, for example, in Beck [1999]. Therefore, studying nonlinear dynamical systems from an experimental point of view and using a physics based methodology and terminology should contribute to help to experimental researchers to a better understanding of this subject.

The more representative paradigm of discrete dynamical systems is the Mandelbrot set [Mandelbrot, 1980, 1983] which is, above all, a mathematical body. As is well known, the Mandelbrot set can be defined by

$$
\mathbf{M}=\left\{c \in \mathrm{C}: f_{c}^{k}(0) \nrightarrow \infty \text { as } k \rightarrow \infty\right\},
$$

where $f_{c}^{k}(0)$ is the $k$-iteration of the complex polynomial function depending on the parameter $c, f_{c}(z)=$ $z^{2}+c, z$ and $c$ complex, for the initial value $z=0$. From the beginning, the Mandelbrot set was amply studied by a great number of researchers. For a first approach to this topic, we could point out either Peitgen \& Richter [1986] and Branner [1989]. Likewise, we should not forget neither the work of Douady and Hubbard [1985] nor that of Milnor [1989]. The complexity of the Mandelbrot set is so big that, even though it has been thoroughly explored, there are always interesting zones to be known and interpreted. Thus, for example, Stephenson [1992, 1994] studies the formulae for cycles and spirals in the Mandelbrot set, or Keller [2000] shows us the "abstract" Mandelbrot set.

By definition, a hyperbolic component of period $p$ in the Mandelbrot set is a connected component of the open set consisting of all parameter values $c$ such that $f_{c}$ has a (necessarily unique) attracting orbit of period $p$ [Milnor, 2000]. Several papers ([Gilbert \& Riordan, 1961], [Metropolis et al., 1973], [May, 1976],

\footnotetext{
${ }^{*}$ This work was published in Int. J. Bifurcation and Chaos 13/8 (2003) 2279-2300
} 
[Lutzky, 1993], [Hao \& Xie, 1993] and [Xie \& Hao, 1994]) studied the number of hyperbolic components in both one-dimensional quadratic maps and the Mandelbrot set, but without dealing with their ordering.

The ordering of the hyperbolic components of the Mandelbrot set began before the set was discovered [Mandelbrot, 1980]. Indeed, the periodic orbits ordering in the real quadratic map $x_{n+1}=x_{n}^{2}+c$ is the same as the hyperbolic component ordering in the Mandelbrot set antenna, and, as it is known, the periodic orbits ordering in quadratic maps was previously studied by Myrberg [1963], Sharkovsky [1964] (see [Sharkovsky et al., 1993]) and Metropolis et al. [1973]. More recently, other authors have studied the partial ordering of the hyperbolic components of the Mandelbrot set by using, for example, "internal addresses" [Lau \& Schleicher, 1994], "rotation numbers" [Devaney, 1997], "R2 naming system" [Munafo, 1999] and "orbit portraits" [Milnor, 2000].

In this work we introduce the notion of "shrub" to study a partial ordering of the structure of the Mandelbrot set. We obtain formulas to calculate the period of the hyperbolic component representative of each branch of a shrub, and the preperiod and period of each Misiurewicz point that is a node or a tip of a shrub. Our ordering is more physics orientated than mathematical; for example, if we choose a Mandelbrot set filament, we would like to know the ordering, if there is an ordering, of the hyperbolic components on such a filament and simple rules to determine their periods.

We associate each hyperbolic component with its period (and each Misiurewicz point with its preperiod and period) in the same way as we did in a previous paper in the case of 1D quadratic maps [Pastor et $a l ., 1996 \mathrm{a}]$. As we can see, the study of this paradigmatic case of dynamical system has been undertaken by mathematicians for the most part. To our way of thinking, a greater contribution of the physicists is desirable in order to have an effect on those aspects with an important value for us, as is the case of the ordering.

The complexity of the Mandelbrot set is so extraordinary that any attempt to order its hyperbolic components must necessarily be partial, above all when the approach is completely experimental as in our case. We shall use only the main tool of a physicist, the measure, instead of the mathematician's tools. Since we have no demonstration, our statements are only valid if there are no new measure that shows the contrary.

Misiurewicz points, [Misiurewicz \& Nitecki, 1991], are omnipresent in the ordering of the hyperbolic components in the same way as in the one-dimensional quadratic maps case [Pastor et al., 1996b, 1997, 1998, 2001], [Romera et al., 1996, 1998]. We name the Misiurewicz points as $\mathbf{M}_{n^{*}, p^{*}}$, where $n^{*}$ is the preperiod and $p^{*}$ the period.

Let $\mathbf{M}_{h c}$ be the set of all the Mandelbrot set hyperbolic components. We name primary hyperbolic components of the Mandelbrot set those directly attached to the main cardioid [Devaney, 1995]. We can associate a rational number $\frac{q}{p}$, the rotation number, to each primary hyperbolic component, and we can use this rotation number to identify each primary hyperbolic component. The rotation number is related to the angle $\alpha$ which varies from 0 to $2 \pi$ when starting from the cusp it turns counter-clockwise to come back to the cusp (see Fig. 1 where the Mandelbrot set is shown). Thus, if several hyperbolic components have the same denominator (the same period), they are ordered in such a way that we find growing values of $q$ when $\alpha$ increases.

We name family of a primary hyperbolic component, $\mathbf{F}\left(\frac{q}{p}\right)$, the set of all the hyperbolic components that are born in such a primary hyperbolic component. The family $\mathbf{F}\left(\frac{q}{p}\right)$ has the following property: to go from any component of the family to the main cardioid, it is necessary to cross the primary hyperbolic component $\frac{q}{p}$. The union of all the primary hyperbolic component families includes all the Mandelbrot set hyperbolic components except for the main cardioid. If we represent the main cardioid by $\mathrm{C}$, we could make a first partition of the Mandelbrot set hyperbolic components as the union of the following disjoint subsets:

$\mathbf{M}_{h c}=\mathrm{C} \cup \mathbf{F}\left(\frac{1}{2}\right) \cup \mathbf{F}\left(\frac{1}{3}\right) \cup \mathbf{F}\left(\frac{2}{3}\right) \cup \mathbf{F}\left(\frac{1}{4}\right) \cup \mathbf{F}\left(\frac{3}{4}\right)$ 
$\cup \mathbf{F}\left(\frac{1}{5}\right) \cup \mathbf{F}\left(\frac{2}{5}\right) \cup \mathbf{F}\left(\frac{3}{5}\right) \cup \mathbf{F}\left(\frac{4}{5}\right) \cup \cdots$

that we represent as

$$
\mathbf{M}_{h c}=\mathrm{C} \cup\left\{\mathbf{F}\left(\frac{q}{p}\right)\right\}
$$

Now, if we want to continue ordering the Mandelbrot set hyperbolic components, we have to order the hyperbolic components of each one of the families. But before undertaking this ordering, we still need to introduce new definitions that we shall see next.

As we saw before, Fig. 1 shows the Mandelbrot set. The end of the period-doubling cascade of any hyperbolic component $h c$ is its Myrberg-Feigenbaum point $\operatorname{MF}(h c)$. What emerges from $\operatorname{MF}(h c)$ is what we denominate a shrub, due to its shape. If the shrub emerges from a primary hyperbolic component $\frac{q}{p}$, then we have a primary shrub, the shrub $\left(\frac{q}{p}\right)$. In Fig. 1 we have framed with a rectangle the primary shrubs emerging from the primary hyperbolic components $\frac{1}{3}(\mathrm{~A}), \frac{1}{4}(\mathrm{~B})$ and $\frac{1}{5}(\mathrm{C})$. These shrubs are enlarged in the left lower part of the figure, and sketches of such shrubs are depicted in the left upper part.

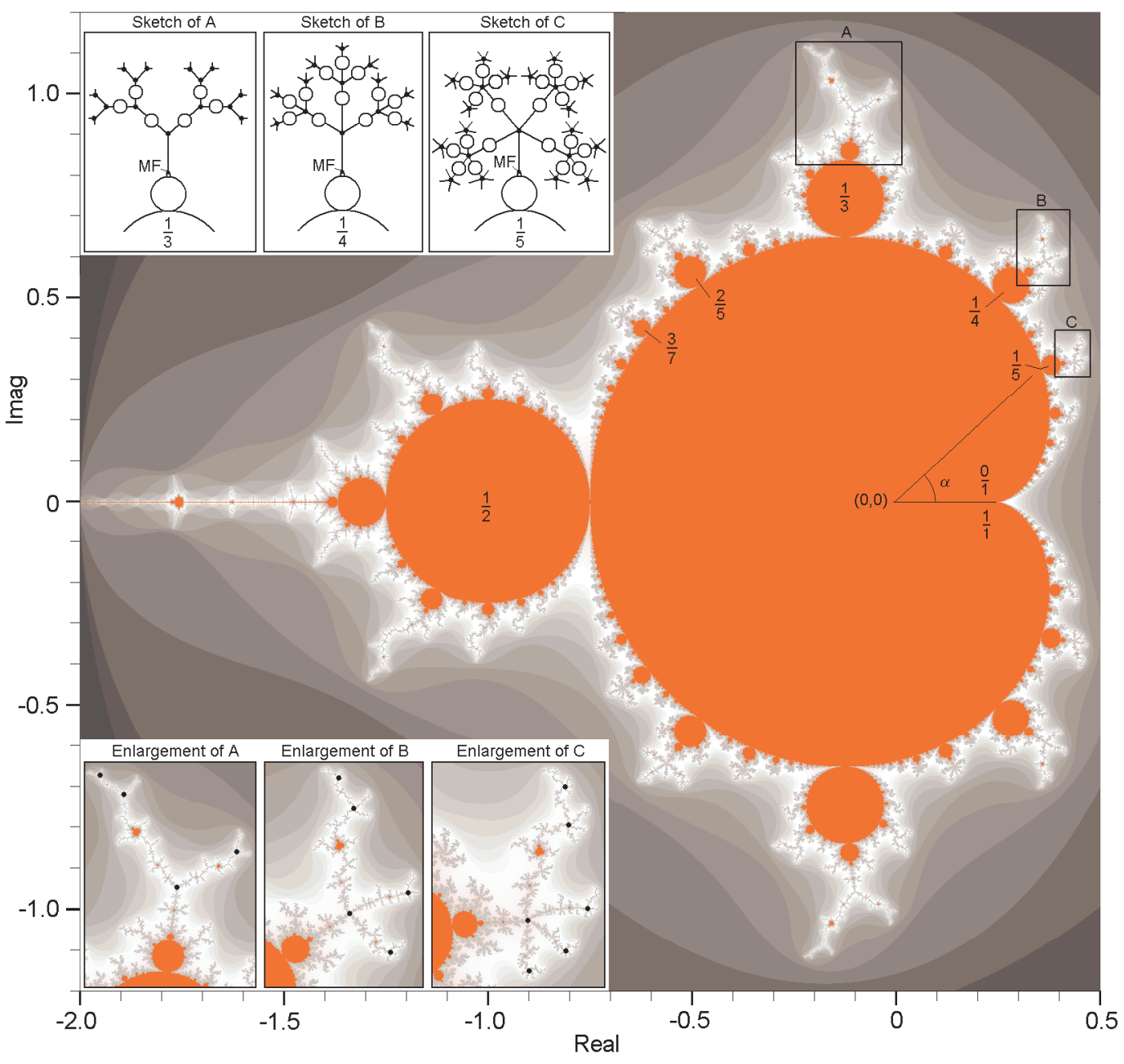

Figure 1: Mandelbrot set. The angle $\alpha(0 \leq \alpha \leq 2 \pi)$ is related to the rotation number $\frac{q}{p}\left(\frac{0}{1} \leq \frac{q}{p} \leq \frac{1}{1}\right)$. Three primary shrubs, shrub $\left(\frac{1}{3}\right)$, shrub $\left(\frac{1}{4}\right)$ and shrub $\left(\frac{1}{5}\right)$ are marked with the rectangles A, B and C. They correspond to the disks with rotation numbers $\frac{1}{3}, \frac{1}{4}$ and $\frac{1}{5}$. Their enlargements and their sketches are shown. 
Now, we can follow a parallel reasoning to that of Douady [1986] when he introduces the tuning method. Attached to each one of the primary hyperbolic components, there is an infinity of secondary hyperbolic components. From the MF point of each one of these hyperbolic components another shrub emerges, which we call a secondary shrub. Likewise, from the MF point of any of the infinite tertiary hyperbolic components, which are attached to each one of the infinite secondary hyperbolic components, another shrub emerges, a tertiary shrub, and so on.

Each family $\mathbf{F}\left(\frac{q}{p}\right)$ has two well differentiated regions: the periodic region and the chaotic region. The periodic region is made up of the primary hyperbolic component $\frac{q}{p}$ and all the $N$-ary $(2 \leq N \leq \infty)$ hyperbolic components whose origin is $\frac{q}{p}$. The chaotic region is made up of an infinity of hyperbolic components mounted on an infinity of shrub branches in each one of the infinity shrubs of the family. Since the ordering in the periodic region has no difficulty, we shall study here the ordering in the chaotic region by using the former introduced shrubs. We shall begin with the most prominent of these shrubs, the primary shrubs, and in the following sections we shall study the secondary, tertiary, etc. shrubs.

\section{Primary Shrubs}

\subsection{Hyperbolic components ordering}

Primary shrubs are associated to a "decoration" formed by a node (see Fig. 1, where the nodes are marked with black points in both the enlarged shrubs and their sketches) from which a number of structural branches [Pastor et al., 1997] emanate. Since all the branches studied in this paper are structural branches, we simply denominate them branches. As is well known, we may read off the period of the primary hyperbolic component by counting the branches of the shrub attached to this decoration. In the left upper part of Fig. 1, we can see that 3,4 and 5 branches emanate from each node of, respectively, shrub $\left(\frac{1}{3}\right)$, $\operatorname{shrub}\left(\frac{1}{4}\right)$ and shrub $\left(\frac{1}{5}\right)$. For our convenience, we divide the set of the $\frac{q}{p}$ valid values into two disjoined subsets: $\frac{1}{p}$, and the rest that we represent by $\frac{q \neq 1}{p}$. Let us begin by studying the primary shrubs of the subset $\frac{1}{p}$.

Figures 2(a), 2(b) and 2(c) show the shrubs of the primary hyperbolic components $\frac{1}{2}$, $\frac{1}{3}$ and $\frac{1}{5}$ (note that Figs. 2(b) and 2(c) correspond to A and C of Fig. 1), and Figs. 3(a), 3(b) and 3(c) show the sketches of these primary shrubs. Fig. 2(a) is on the real axis and Figs. 2(b) and 2(c) are in the upper part of the Mandelbrot set. Note that all what we say for this upper part also holds for the lower part because the Mandelbrot set is symmetric with regard to the real axis.

To begin with, let us see shrub $\left(\frac{1}{3}\right)$ in Fig. 2(b) and its sketch in Fig. 3(b). We associate a number (the associated number) with each branch of the shrub and its corresponding node. We name main branch the branch that emerges from the MF point. We associate the number 0 to the main branch because, as we shall see later, this branch does not belong to the shrub. The main branch finishes at the main node (whose associated number is also 0) where the branches of the first level begin; and, therefore the main node is the point where in fact the shrub begins. Since from each node emanate three branches and one is the main branch, in the first level there are two branches. Starting from the main branch 0 we turn clockwise around the main node, and we name the two $\left(2^{1}\right)$ branches of the first level as 1 and 2 by following the branches one by one. Each one of these two branches finishes at one of the two nodes of the first level (each one of them associated to the number of its corresponding branch). Starting from any of the two branches of the first level, and turning clockwise around any of the two nodes of this first level, we obtain two new branches that we name as the number of the first level origin branch and by adding a 1 to the first one and a 2 to the second one following the branches one by one; i. e., if we start from 1 we obtain 11 and 12 , and if we start from 2 we obtain 21 and 22 . In such a manner, we have obtained the four branches $\left(2^{2}\right)$ of the second level, which finish at the four corresponding nodes of the second level, and so on, as can be seen in Fig. 3(b). Therefore, we associate a number with $m$ digits to each branch of the level $m$ (and to its corresponding node). For the shrub $\left(\frac{1}{3}\right)$ case, the level $m$ has $(3-1)^{m}$ branches of $m$ digits that finish 
at $(3-1)^{m}$ nodes of $m$ digits.

Let us see now shrub $\left(\frac{1}{5}\right)$ in Fig. 2(c) and its sketch in Fig. 3(c). Starting from the main branch 0 we go to the first level branches 1, 2, 3 and 4, by following the same direction and following branches one by one as in the previous case. Therefore, the first level has now four branches $\left(4^{1}\right)$ of 1 digits. We obtain the branches of the second level by adding again 1,2,3 and 4, in the same way as before, to each one of the branches of the previous level. Hence, the second level has sixteen branches $\left(4^{2}\right)$ of 2 digits, and so on. Hence, for the case of the shrub $\left(\frac{1}{5}\right)$, the level $m$ has $(5-1)^{m}$ branches of $m$ digits.

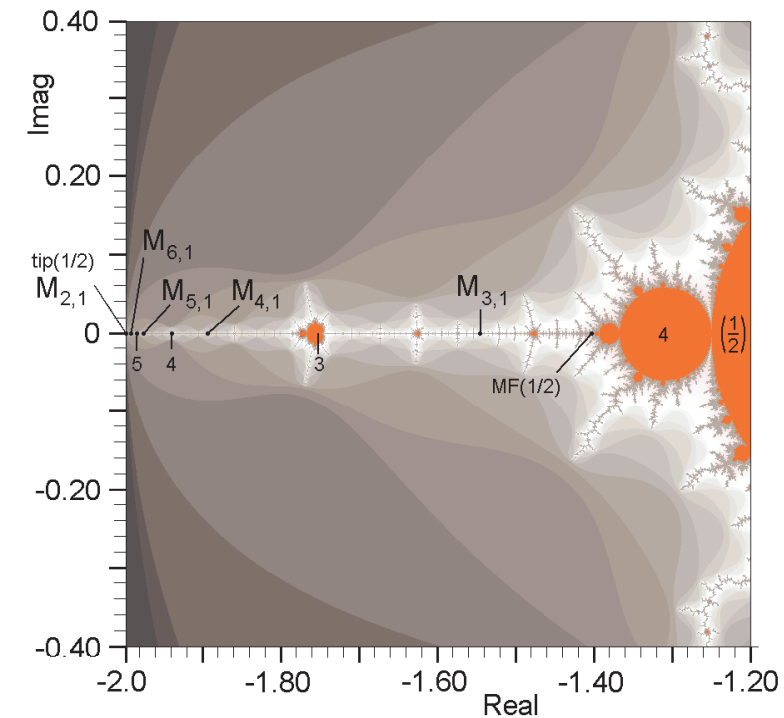

(a)

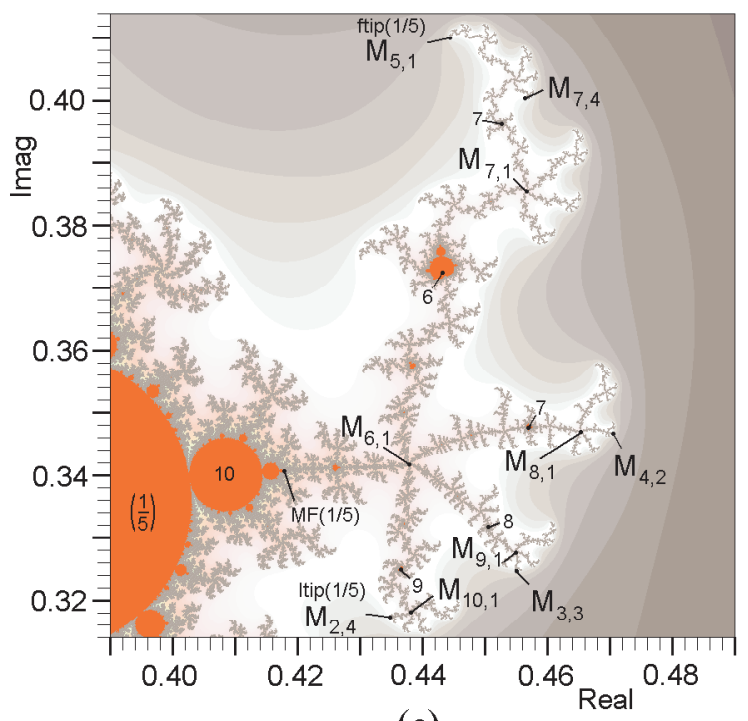

(c)

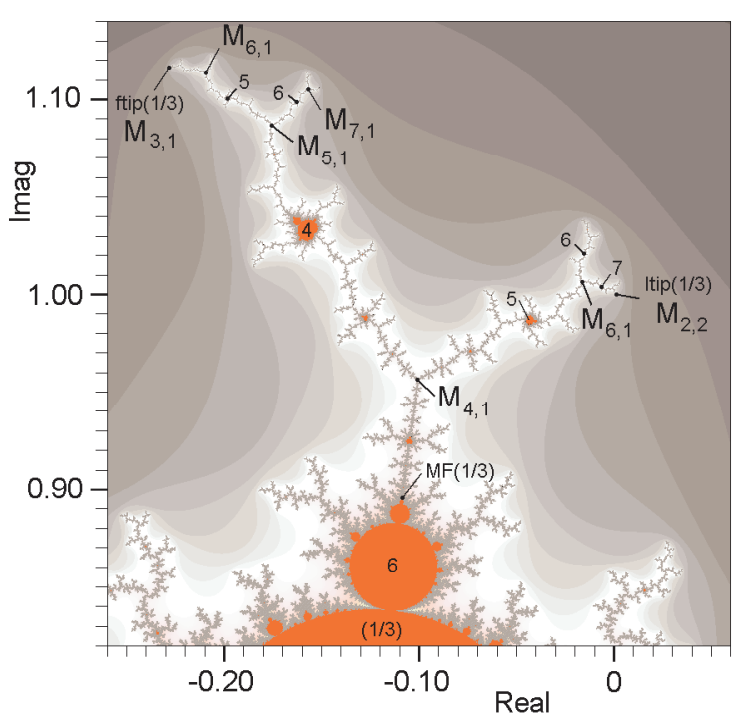

(b)

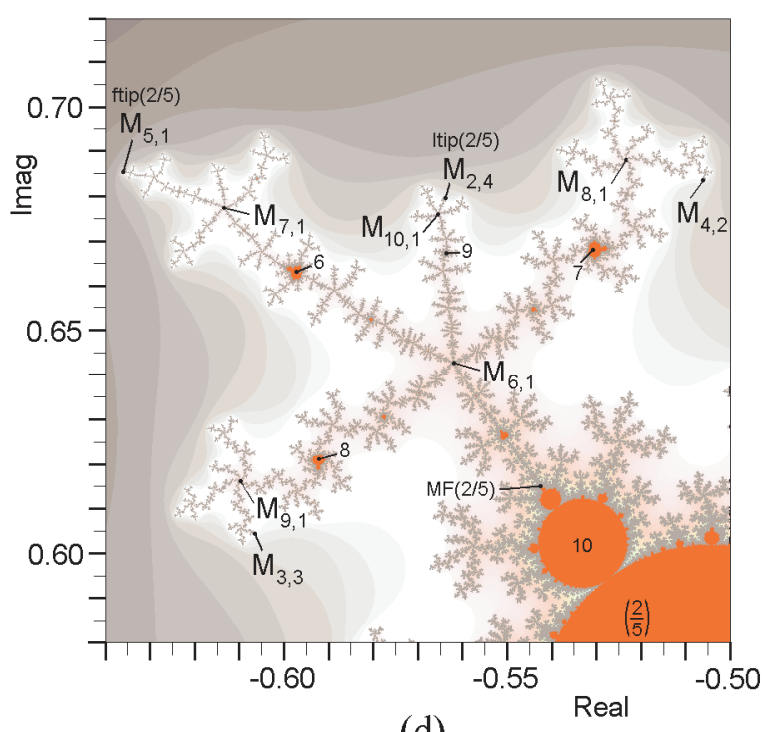

(d)

Figure 2: Mandelbrot set regions showing four primary shrubs. a) shrub $\left(\frac{1}{2}\right)$. b) shrub $\left(\frac{1}{3}\right)$. c) shrub $\left(\frac{1}{5}\right)$. d) $\operatorname{shrub}\left(\frac{2}{5}\right)$.

Therefore, we can generalise for the case of shrub $\left(\frac{1}{p}\right)$. Its main branch is the 0 , which finishes in the main node. In the first level we have the branches $1,2, \ldots,(p-1)$. In this level we always have one digit, and if $p \geq 10$ we have to use, for example, Greek letters or other symbols to indicate $10,11, \ldots$ with only one digit. In the first level, we have then $(p-1)^{1}$ branches of 1 digit. In the second level we have $(p-1)^{2}$ 
branches of 2 digits, and so on. Therefore, for the case of the shrub $\left(\frac{1}{p}\right)$, the level $m$ has $(p-1)^{m}$ branches of $m$ digits.

Let us note that in Figs. 2(a) and 3(a), which represents the shrub $\left(\frac{1}{2}\right)$, we apparently have only one branch. This is because the first level has only one branch, the same as the second level and all the others. Indeed the main branch is the branch 0 , in the first level we have again only one branch, $(2-1)^{1}=1$, the branch 1 , in the second level again only one branch, $(2-1)^{2}=1$, the branch 11 , and so on, obtaining a sequence of successive branches giving as a result an apparently unique branch. Let us see now the case

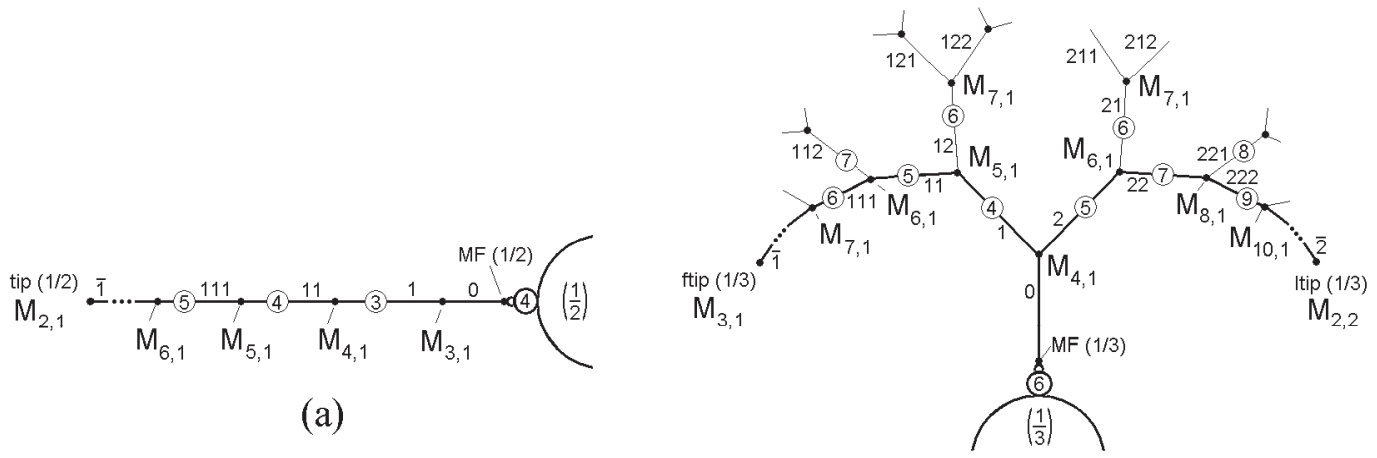

(b)

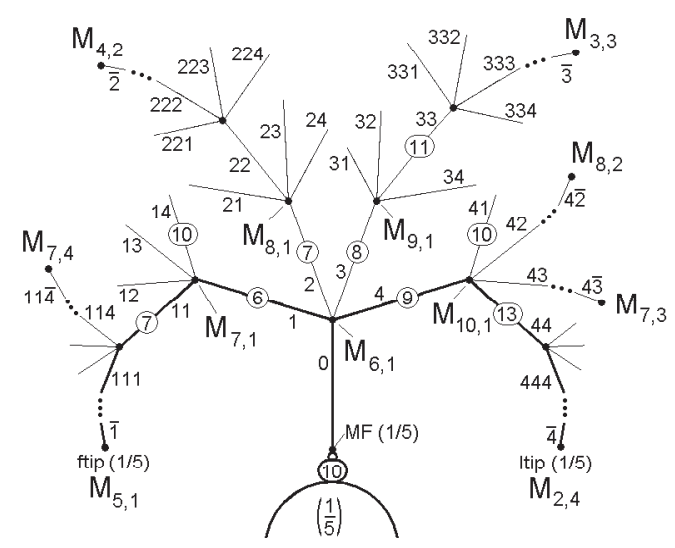

(c)

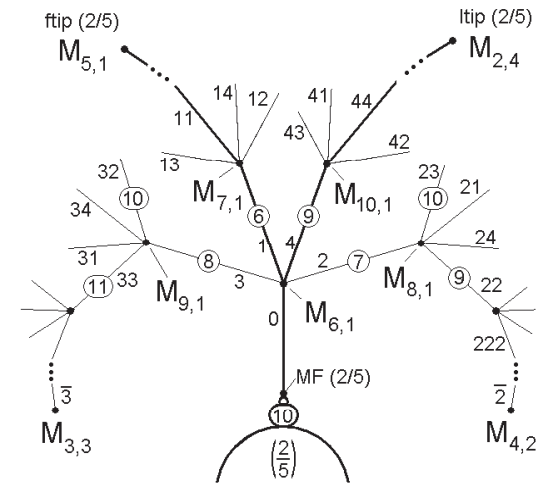

(d)

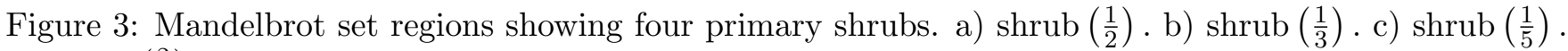
d) $\operatorname{shrub}\left(\frac{2}{5}\right)$.

of a rotation number with numerator different to 1 ; i. e., the case $\frac{q \neq 1}{p}$. Figure $2(\mathrm{~d})$ shows shrub $\left(\frac{2}{5}\right)$, and Fig. 3(d) shows a sketch of shrub $\left(\frac{2}{5}\right)$. We name 0 the main branch. In the first level we also have four branches, 1, 2, 3 and 4 , as in the shrub $\left(\frac{1}{5}\right)$, but now they are placed in a different way. When $q=1$, we number them by turning clockwise around the main node branch by branch. Now, when $q=2$, we number the branches of the first level also by turning clockwise around the main node but now two by two, as can be seen in Fig. 3(d). The same happens for the following levels. In general, when the numerator of the rotation number is $q \neq 1$ we number the branches of the first level by turning clockwise around the main node $q$ by $q$ branches. We have the same for the following levels.

In each branch of any shrub there are an infinity of hyperbolic components. We denominate hyperbolic component representative of this branch, or simply representative, the hyperbolic component with the smallest period in this branch (which is also that with the biggest size). We have experimentally seen that the period of the representative $p_{r}$ of any level $m$ branch of a primary shrub $\left(\frac{q}{p}\right)$, is given by

$$
p_{r}=p+\Sigma,
$$


where $\Sigma=d_{1}+\cdots+d_{m}$ is the sum of the $m$ digits of the branch associated number $d_{1} d_{2} \cdots d_{m}$. Thus, the period of the representative of the branch 221 in the third level of shrub $\left(\frac{1}{3}\right)$ is 8 , see Fig. 3(b), the period of the representative of the branch 33 in the second level of shrub $\left(\frac{1}{5}\right)$ is 11, see Fig. 3(c), and the period of the representative of the branch 33 in the second level of shrub $\left(\frac{2}{5}\right)$ is 11 , see Fig. $3(\mathrm{~d})$. In last both cases we obtain the same period value, but the corresponding representatives are located in different places.

In the case of shrub $\left(\frac{1}{2}\right)$, Figs. 2(a) and 3(a), when we start from the main branch 0 and we go to the first, second, third, ... levels we find the branches $1,11,111, \ldots$ and so on until the infinity where we reach the end, which we name tip $\left(\frac{1}{2}\right)$. This is the only tip, as opposed to all the other cases. Indeed, in shrub $\left(\frac{1}{3}\right)$, see Fig. 3(b), starting also from the branch 0, now we have an infinity of different paths that lead us to an infinity of different ends. Therefore, we have an infinity of tips. But among all these possibilities we are going to point out two paths and their two ends. The first one is that which we name first path which is given by the branches $1,11,111, \ldots$ and its end is what we name first tip or ftip $\left(\frac{1}{3}\right)$. The second one is what we name last path, which is given by the branches $2,22,222, \ldots$, and its end is what we name last tip or ltip $\left(\frac{1}{3}\right)$. In all the cases ftip $\left(\frac{q}{p}\right)$ is given by the branches $1,11,111, \ldots$, while ltip $\left(\frac{q}{p}\right)$ is given by the branches $(p-1),(p-1)(p-1),(p-1)(p-1)(p-1) \ldots$ These two singular paths have been marked with a thick line in the shrubs of Fig. 3.

In the case of the shrub $\left(\frac{1}{p}\right)$, "first path" and "last path" correspond to their geometrical mean, since they are at the outermost ends, and all the others are in between. However, in the case of the shrub $\left(\frac{q \neq 1}{p}\right)$, the geometrical meaning is lost and they are no longer at the extremes.

\subsection{Misiurewicz points ordering}

Shrubs have two special types of points: nodes and tips. The first ones separate the different levels of branches and the second ones are the ends of the branch paths. Let us point out that both, nodes and tips, are Misiurewicz points.

As can be deduced from the experimental results shown in Fig. 3, all the nodes in a primary shrub are characteristic Misiurewicz points because they have period one [Romera et al., 1996]. In addition, the preperiod of a node of level $m$ can be calculated from its associated number $d_{1} d_{2} \ldots d_{m}$. Indeed, a node of level $m$ belonging to shrub $\left(\frac{q}{p}\right)$ is a characteristic Misiurewicz point $\mathrm{M}_{n^{*}, p^{*}}$ with preperiod $n^{*}$ and period $p^{*}$ given by

$$
n^{*}=p+\Sigma+1=p_{r}+1, p^{*}=1,
$$

where $\Sigma=d_{1}+\cdots+d_{m}$ is the sum of the $m$ digits of the node associated number, and $p_{r}$ is the period of the representative of the branch where the node is.

Let us see now tips in Fig. 3(c). If we follow the path $1,11,111, \ldots$, we reach the tip $\mathrm{M}_{5,1}$; if we follow the path $2,22,222, \ldots$, we reach the tip $\mathrm{M}_{4,2}$; if we follow the path $3,33,333, \ldots$, we reach the tip $\mathrm{M}_{3,3}$; and, finally, if we follow the path $4,44,444, \ldots$, we reach the tip $\mathrm{M}_{2,4}$. We call these paths unlocked paths (u.p.) because through these paths we can reach a tip. Let us note that in unlocked paths the associated number of each new branch adds always a same digit $d$. According to these results, we can say that if we start from any primary hyperbolic component $\frac{q}{p}$ and we follow an unlocked path, at the infinity we reach a tip that is a Misiurewicz point $\mathrm{M}_{n^{*}, p^{*}}$ with

$$
n^{*}=p-d+1, p^{*}=d .
$$

Indeed, in the former four examples $p=5$ and $d=1,2,3$ or 4 ; therefore, according to Eq. (5) the tips are the Misiurewicz points $\mathrm{M}_{5,1}, \mathrm{M}_{4,2}, \mathrm{M}_{3,3}$ and $\mathrm{M}_{2,4}$.

We can consider two particular cases of Eq. (5). First, if we follow the first path $1,11,111, \ldots$, then $d=1$, we reach the ftip and Eq. (5) becomes

$$
\mathrm{ftip}\left(\frac{q}{p}\right)=\mathrm{M}_{p, 1}
$$


and second, if we follow the last path $(p-1),(p-1)(p-1), \cdots, \overline{p-1}$ then $d=p-1$, we reach the ltip and Eq. (5) becomes

$$
\operatorname{ltip}\left(\frac{q}{p}\right)=\mathrm{M}_{2, p-1} \text {. }
$$

The only path in shrub $\left(\frac{1}{2}\right)$, see Fig. $3(\mathrm{a})$, is at the same time the first path and the last path. Indeed, we obtain $\operatorname{tip}\left(\frac{1}{2}\right)=\mathrm{M}_{2,1}$ in both cases if we apply Eq. (6) for $p=2$, which computes ftip $\left(\frac{1}{2}\right)$, or if we apply Eq. (7) for $p=2$, which computes $\operatorname{ltip}\left(\frac{1}{2}\right)$.

Let us analyze what we did to reach a tip. Starting from the main branch 0 of a primary hyperbolic component $\frac{q}{p}$ we have followed unlocked paths like $2,22, \cdots, \overline{2}$ whose associated numbers are periodic digits $\bar{d}$. But we can reach a tip by mean of an unlocked path with two steps. In the first one, the preperiodic step, we follow the path of $m$ branches $d_{1}, d_{1} d_{2}, \cdots, d_{1} d_{2} \cdots d_{m}$ and we reach the representative of period $p_{r}=p+\Sigma$, where $\Sigma=d_{1}+\cdots+d_{m}$. This first step can be non-existent as in the former cases seen. In the second step, the periodic step, we follow the path $d_{m+1}, d_{m+1} d_{m+1}, \cdots, \overline{d_{m+1}}$ that always adds the same digit $d_{m+1}$. This second step can be thought as the true unlocked path.

In the case of an unlocked path with two steps Eq. (5) is no longer valid. In order to generalise Eq. (5), it seems reasonable to put $p_{r}$ instead of $p$. As we have experimentally obtained, this is so when $\Sigma=0$ or $d_{m}>d_{m+1}$. We shall name this case as the normal case. In the normal case, if we start from any primary hyperbolic component $\frac{q}{p}$ and we follow the path $d_{1}, d_{1} d_{2}, \cdots, d_{1} d_{2} \cdots d_{m}$, $d_{1} d_{2} \cdots d_{m} d_{m+1}, \cdots, d_{1} d_{2} \cdots d_{m} \overline{d_{m+1}}$, at the infinity we reach a tip that is a Misiurewicz point $\mathrm{M}_{n^{*}, p^{*}}$ with

$$
n^{*}=p+\Sigma-d_{m+1}+1, p^{*}=d_{m+1}
$$

where $\Sigma=d_{1}+\cdots+d_{m}$. When $\Sigma=0$, Eq. (8) becomes Eq. (5), which is a particular case of the normal case. Thus, as we can see in Fig. 3(c), if we follow the path $4,42, \cdots, 4 \overline{2}\left(\Sigma=4, d_{m}=4, d_{m+1}=2\right)$ according to Eq. (8) we reach the tip $\mathrm{M}_{8,2}$.

When we are not in the normal case we are in the especial case. We have the especial case when $\Sigma \neq 0 \cap d_{m}<d_{m+1}$. In the especial case Eq. (8) becomes

$$
n^{*}=p+\Sigma-d_{m}+1, p^{*}=d_{m+1} .
$$

Equations (8) and (9) only differ in that $\Sigma-d_{m+1}$ changes to $\Sigma-d_{m}$. Thus, as we can see in Fig. $3(\mathrm{c})$, if we follow the path $1,11,114, \cdots, 11 \overline{4}\left(\Sigma=2, d_{m}=1, d_{m+1}=4\right)$ according to Eq. (9) we reach the tip $\mathrm{M}_{7,4}$. In both normal and especial cases, if $d_{m+1}=1$ the tip is a non-characteristic Misiurewicz point [Romera et al., 1996], and if $d_{m+1}=1$ the tip is a characteristic Misiurewicz point. In both cases the tips are crisis points [Grebogi et al., 1982]. As we shall see later, the more important u.p. occurs when $d_{m+1}=1$, that we shall call a premier unlocked path (p.u.p.). A p.u.p. has also two steps, the preperiodic one $d_{1}, d_{1} d_{2}, \cdots, d_{1} d_{2} \cdots d_{m}$ (can exist or not) and the periodic one $d_{m+1}, d_{m+1} d_{m+1}, \cdots, \overline{d_{m+1}}=\overline{1}$ (always exists). Now, either $\Sigma=0$ or $d_{m}>1$ hence a premier unlocked path is always in the normal case and we have to use Eq. (8) with $d_{m+1}=1$ to calculate its tip. After the second step of a premier unlocked path we reach a premier tip or ptip. In the next section we shall see the importance of ptips.

It is possible to have another type of u.p. as $d_{1}, d_{1} d_{2}, \cdots, d_{1} d_{2} \cdots d_{m} \overline{d_{m+1} \cdots d_{m+i}}$. In this type of paths we reach a tip by following an u.p. whose second step is constituted by a "short cut" with the same group of digits [Pastor et al., 2001]. However, since this does not provide any important contribution, we shall not treat here these cases.

So far, in this section we have studied the primary shrubs, which are the shrubs of the primary hyperbolic components $\frac{q}{p}$. Each one of these shrubs has always the same decoration that is constituted by a node from which $p$ branches emanate. Next, we shall study the secondary shrubs, which are the shrubs of the secondary hyperbolic components $\frac{q_{1}}{p_{1}} \cdot \frac{q_{2}}{p_{2}}$. As we shall see, these shrubs are associated to two decorations, 
the first one a node from which $p_{2}$ branches emanate and the second one a node from which $p_{1}$ branches emanate. That is why we divide the secondary shrubs into two subshrubs, each one of them associated to one of these decorations. Let us see it.

\section{Secondary Shrubs}

We denominate secondary hyperbolic component, $\frac{q_{1}}{p_{1}} \cdot \frac{q_{2}}{p_{2}}$, to the hyperbolic component attached to the primary hyperbolic component $\frac{q_{1}}{p_{1}}$ in the relative position that $\frac{q_{2}}{p_{2}}$ should have with regard to the main cardioid. From MF $\left(\frac{q_{1}}{p_{1}} \cdot \frac{q_{2}}{p_{2}}\right)$ a secondary shrub emerges, shrub $\left(\frac{q_{1}}{p_{1}} \cdot \frac{q_{2}}{p_{2}}\right)$. Let us see what these shrubs look like.

For that, let us see Fig. 4 where a secondary shrub, shrub $\left(\frac{1}{3} \cdot \frac{1}{5}\right)$, is shown. This shrub has two subshrubs. The first one, subshrub $1\left(\frac{1}{3} \cdot \frac{1}{5}\right)$, which is born from $\operatorname{MF}\left(\frac{q_{1}}{p_{1}} \cdot \frac{q_{2}}{p_{2}}\right)$, is related to the component $\frac{q_{2}}{p_{2}}$ (here $\frac{1}{5}$ ). The second one, subshrub $2\left(\frac{1}{3} \cdot \frac{1}{5}\right)$, which is born from each one of the p.u.p. ends of the first subshrub is related to the component $\frac{q_{1}}{p_{1}}$ (here $\frac{1}{3}$ ). Since a secondary shrub is a complex structure, let us analyse shrub $\left(\frac{1}{3} \cdot \frac{1}{5}\right)$ very carefully by using the sketch of Fig. 5. In this figure we have separate $\operatorname{subshrub}_{1}\left(\frac{1}{3} \cdot \frac{1}{5}\right)$ and subshrub $2\left(\frac{1}{3} \cdot \frac{1}{5}\right)$. It is not easy to see the correspondence between the shrub and its sketch (Figs. 4 and 5). Indeed, some branches of a same level larger than others in Fig. 4 but they have been depicted as equals in Fig. 5. As we can see in the sketch of Fig. 5, we can reach a tip of the first subshrub in a similar way as we did for primary shrubs, i.e., by following an u.p. $d_{11}, d_{11} d_{12}, \cdots, d_{11} d_{12} \cdots d_{1 m_{1}} \overline{d_{1\left(m_{1}+1\right)}}$. Indeed, first we follow a first step $d_{11}, d_{11} d_{12}, \cdots, d_{11} d_{12} \cdots d_{1 m_{1}}$ of this u.p. until a level $m_{1}$ representative $p_{r_{1}}$. This first step can exist or not. And finally, starting from $p_{r_{1}}$ we follow the second step $\overline{d_{1\left(m_{1}+1\right)}}$ to reach the tip.

Note that if we are in the first subshrub of a secondary shrub, we only can reach the second subshrub by following a p.u.p., i.e. only if $d_{1\left(m_{1}+1\right)}=1$. When $d_{1\left(m_{1}+1\right)} \neq 1$ we reach a tip that is a crisis point and the second subshrub is not reached (obviously, a ptip is not a crisis point). We have an infinity of paths to reach the second subshrub. Hence, the second subshrub has an infinity of separated portions, which are non-connected, each one emerging from a ptip of the first subshrub. In Fig. 5 we mark with a little black circle the ends of the p.u.p. (ptips) and with a little black square the ends of the other u.p.. From each ptip, a portion of the second subshrub emerges. In Fig. 5 we show three of these infinite portions of the second subshrub, those that come from the end of the p.u.p. 1,11, $\cdots, \overline{1}\left(\mathrm{M}_{13,1}\right), 3,31, \cdots, 3 \overline{1}\left(\mathrm{M}_{22,1}\right)$ and $4,41, \cdots 4 \overline{1}\left(\mathrm{M}_{25,1}\right)$.

If we are in a portion of the second subshrub, we can reach a tip by following an u.p. $d_{21}, d_{21} d_{22}, \cdots, d_{21} d_{22} \cdots d_{2 m_{2}} \overline{d_{2\left(m_{2}+1\right)}}$ with two steps: the first step $d_{21}, d_{21} d_{22}, \cdots, d_{21} d_{22} \cdots d_{2 m_{2}}$, that can exist or not, until a level $m_{2}$ and the second step $\overline{d_{2\left(m_{2}+1\right)}}$ to reach the tip. Since we are in the last subshrub, whether the u.p. is a p.u.p. or not, either ptips and tips are crisis points. Let us see both subshrubs.

\subsection{First subshrub hyperbolic components ordering}

Let us consider a secondary shrub $\left(\frac{q_{1}}{p_{1}} \cdot \frac{q_{2}}{p_{2}}\right)$, as shrub $\left(\frac{1}{3} \cdot \frac{1}{5}\right)$ of Fig.5. We can see that five branches emanate from each node of the first subshrub. We assign associated numbers to the branches of the first subshrub. Thus, the four branches of the first level are 1,2,3 and 4, the sixteen branches of the second level (the associated numbers are not shown in the figure) are 11, 12, 13, 14, 21, 22, 23, 24, 31, 32, 33, 34, 41, 42, 43 and 44 , and so on. We can again experimentally determine the period of every representative of a branch $d_{11} d_{12} \cdots d_{1 m_{1}}$ of the first subshrub that is given by

$$
p_{r_{1}}=p_{1}\left(p_{2}+\Sigma_{1}\right)
$$




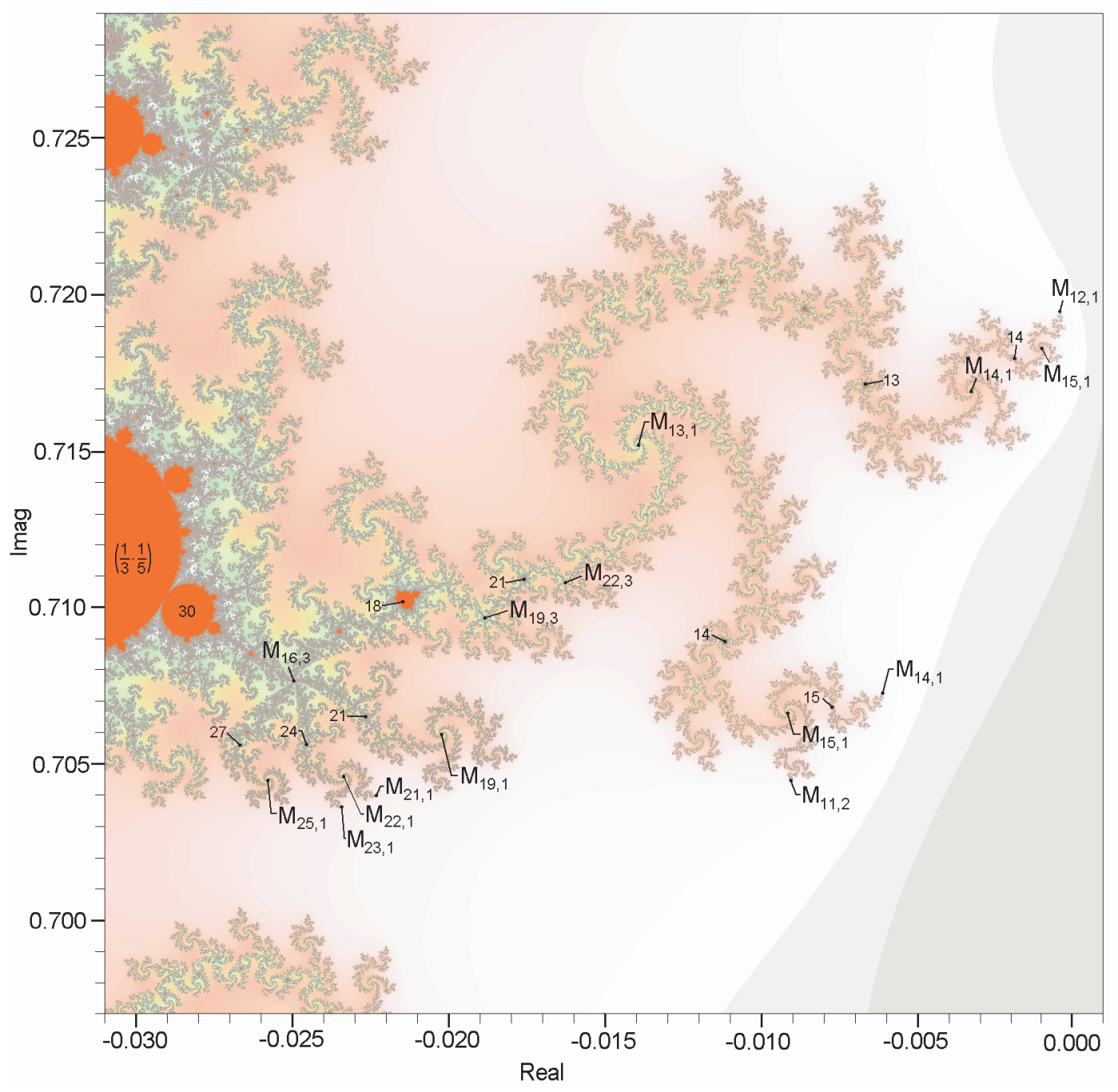

Figure 4: Mandelbrot set region showing a secondary shrub, shrub $\left(\frac{1}{3} \cdot \frac{1}{5}\right)$

where $\Sigma_{1}=d_{11}+d_{12}+\cdots+d_{1 m_{1}}$. Thus, the periods of the representatives of the branches 1,21 and 42 of $\operatorname{subshrub}_{1}\left(\frac{1}{3} \cdot \frac{1}{5}\right)$ are 18,24 and 33 .

\subsection{Second subshrub hyperbolic components ordering}

We indicate that we have left the first subshrub and we have reached the main node of a portion of the second subshrub by adding one arrow to the p.u.p. that we have followed. Thus, in Fig. 5 we have reached three main nodes: $\overline{1} \rightarrow, 3 \overline{1} \rightarrow$ and $4 \overline{1} \rightarrow$ the Misiurewicz points $\mathrm{M}_{13,1}, \mathrm{M}_{22,1}$ and $\mathrm{M}_{25,1}$. We shall denominate any portion with the same notation as with its main node.

All the portions of the subshrub $2\left(\frac{1}{3} \cdot \frac{1}{5}\right)$ have three branches around each node except the main one (we have not branch 0). Again, we name each branch with its associated number. Therefore, in the first level of any portion we have two branches (1 and 2), in the second level four branches (11, 12 and 21, 22), and so on. But in order to distinguish the branches of a portion from the branches of another portion, we must indicate first the portion notation. Thus, the branches emanating from the portion on the left of Fig. 5 are $\overline{1} \rightarrow 1$ and $\overline{1} \rightarrow 2$, from the portion on the centre are $3 \overline{1} \rightarrow 1$ and $3 \overline{1} \rightarrow 2$, and from the portion on the right are $4 \overline{1} \rightarrow 1$ and $4 \overline{1} \rightarrow 2$. These are the complete associated numbers, while the previous ones were the local associated numbers or simply associated numbers. We can experimentally determine the period 


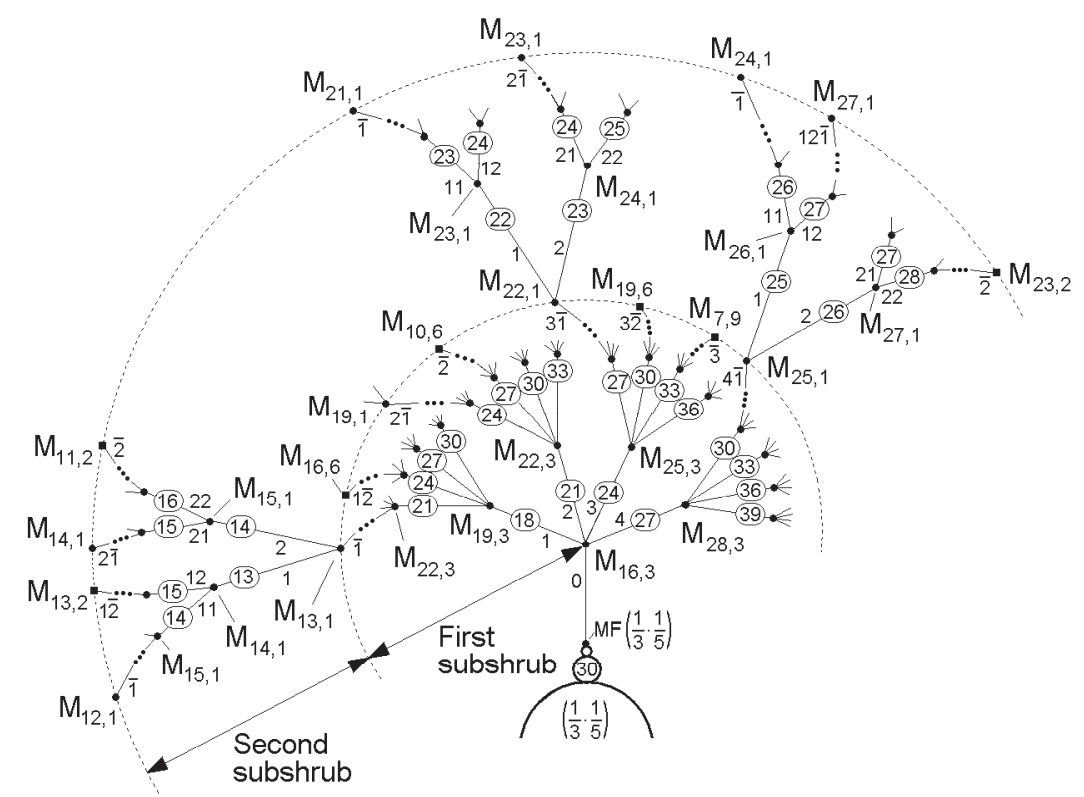

Figure 5: Sketch of the secondary shrub of Fig. 4, shrub $\left(\frac{1}{3} \cdot \frac{1}{5}\right)$, and its two subshrubs. The second subshrub has an infinity of portions, and three of them are depicted on the figure (those coming from $\overline{1}$, $3 \overline{1}$ and $4 \overline{1})$.

of the representative of the branch $d_{11} d_{12} \cdots d_{1 m_{1}} \overline{d_{1\left(m_{1}+1\right)}} \rightarrow d_{21} d_{22} \cdots d_{2 m_{2}}$ of subshrub $\left(\frac{q_{1}}{p_{1}} \cdot \frac{q_{2}}{p_{2}}\right)$ that is given by

$$
p_{r_{2}}=p_{1}\left(p_{2}+\Sigma_{1}-1\right)+\Sigma_{2},
$$

where $\Sigma_{1}=d_{11}+d_{12}+\cdots+d_{1 m_{1}}$ and $\Sigma_{2}=d_{21}+d_{22}+\cdots+d_{2 m_{2}}$. Figure 5 shows three portions of $\operatorname{subshrub}_{2}\left(\frac{1}{3} \cdot \frac{1}{5}\right)$. The first one is $\overline{1} \rightarrow$ with $\Sigma_{1}=0$. In this portion the periods of the representatives of the branches $\overline{1} \rightarrow 11$ and $\overline{1} \rightarrow 21$ are 14 and 15 . The second one is $3 \overline{1} \rightarrow$ with $\Sigma_{1}=3$, and the periods of the representatives of the branches $3 \overline{1} \rightarrow 12$ and $3 \overline{1} \rightarrow 22$ are 24 and 25 . The third one is $4 \overline{1} \rightarrow$ with $\Sigma_{1}=4$, and the periods of the representatives of the branches $4 \overline{1} \rightarrow 12$ and $4 \overline{1} \rightarrow 21$ are 27 and 27 .

Let us study the nodes and tips of the two subshrubs of a secondary shrub. We begin with the first subshrub.

\subsection{First subshrub Misiurewicz points ordering}

In accordance with our experimental data, a node $d_{11} d_{12} \cdots d_{1 m_{1}}$ of the $\operatorname{subshrub}_{1}\left(\frac{q_{1}}{p_{1}} \cdot \frac{q_{2}}{p_{2}}\right)$ is a noncharacteristic Misiurewicz point $\mathrm{M}_{n^{*}, p^{*}}$ with

$$
n^{*}=p_{1}\left(p_{2}+\Sigma_{1}\right)+1, p^{*}=p_{1},
$$

where $\Sigma_{1}=d_{11}+d_{12}+\cdots+d_{1 m_{1}}$. Thus, the nodes $1,2,3$ and 4 of $\operatorname{subshrub}_{1}\left(\frac{1}{3} \cdot \frac{1}{5}\right)$ of Fig. 5 are the non-characteristic Misiurewicz points $\mathrm{M}_{19,3}, \mathrm{M}_{223}, \mathrm{M}_{25,3}, \mathrm{M}_{28,3}$. If we compare Eqs. (10) and (12), we can see that the preperiod of a level $m_{1}$ node of $\operatorname{subshrub}_{1}\left(\frac{q_{1}}{p_{1}} \cdot \frac{q_{2}}{p_{2}}\right)$ is $p_{r_{1}}+1$.

Let us see now tips. To reach a tip we have to follow an u.p. $d_{11}, d_{11} d_{12}, \cdots, d_{11} d_{12} \cdots d_{1 m_{1}} \overline{d_{1\left(m_{1}+1\right)}}$. Let $\Sigma_{1}=d_{11}+d_{12}+\cdots+d_{1 m_{1}}$. As in primary shrubs, we have again a normal case and an especial case.

When $\Sigma_{1}=0 \cup d_{1 m_{1}}>d_{1\left(m_{1}+1\right)}$ we are in the normal case. As can experimentally be seen, in the normal case the tips are Misiurewicz points $\mathrm{M}_{n^{*}, p^{*}}$ with

$$
n^{*}=p_{1}\left(p_{2}+\Sigma_{1}-d_{1\left(m_{1}+1\right)}\right)+1,
$$




$$
p^{*}=p_{1} d_{1\left(m_{1}+1\right)} .
$$

Let us apply Eq. (13) for calculate tips in subshrub $1\left(\frac{1}{3} \cdot \frac{1}{5}\right)$ of Fig. 5. If we follow the paths $2,22, \cdots, \overline{2}$ and $3,32, \cdots, 3 \overline{2}$ we obtain the Misiurewicz points $\mathrm{M}_{10,6}$ and $\mathrm{M}_{19,6}$.

When $\Sigma_{1} \neq 0 \cap d_{1 m_{1}}<d_{1\left(m_{1}+1\right)}$ we are in the especial case and Eq. (13) becomes

$$
n^{*}=p_{1}\left(p_{2}+\Sigma_{1}-d_{1 m_{1}}\right)+1, p^{*}=p_{1} d_{1\left(m_{1}+1\right)} .
$$

Thus, if we follow the path $1,12, \cdots, 1 \overline{2}$ in subshrub $1\left(\frac{1}{3} \cdot \frac{1}{5}\right)$ of Fig. 5 we reach $\mathrm{M}_{16,6}$. Note that Eqs. (13) and (14) only differ in that $\Sigma_{1}-d_{1\left(m_{1}+1\right)}$ changes to $\Sigma_{1}-d_{1 m_{1}}$.

Let us point out an apparent anomaly. As we already know, if we follow a p.u.p., $d_{1\left(m_{1}+1\right)}=1$, we do not reach a crisis point tip but a main node of the second subshrub. A p.u.p. is always in the normal case, hence, we have to apply Eq. (13). However, as we can see in Fig. 5, all the main nodes in the second subshrub (the last subshrub in a secondary shrub) are period-one Misiurewicz points. Hence, Eq. (13) becomes

$$
n^{*}=p_{1}\left(p_{2}+\Sigma_{1}-1\right)+1, p^{*}=1 .
$$

Thus, for subshrub $1\left(\frac{1}{3} \cdot \frac{1}{5}\right)$ of Fig. 5 , if we follow the path $4,41, \cdots, 4 \overline{1}$ we obtain the Misiurewicz point $\mathrm{M}_{25,1}$.

\subsection{Second subshrub Misiurewicz points ordering}

In accordance with our experimental data, a node $d_{11} d_{12} \cdots d_{1 m_{1}} \overline{d_{1\left(m_{1}+1\right)}} \rightarrow d_{21} d_{22} \cdots d_{2 m_{2}}$ of the $\operatorname{subshrub}_{2}\left(\frac{q_{1}}{p_{1}} \cdot \frac{q_{2}}{p_{2}}\right)$ is a characteristic Misiurewicz point $\mathrm{M}_{n^{*}, p^{*}}$ with

$$
n^{*}=p_{1}\left(p_{2}+\Sigma_{1}-1\right)+\Sigma_{2}+1, p^{*}=1 \text {. }
$$

where $\Sigma_{1}=d_{11}+d_{12}+\cdots+d_{1 m_{1}}$ and $\Sigma_{2}=d_{21}+d_{22}+\cdots+d_{2 m_{2}}$. Thus, the node $\overline{1} \rightarrow 11$ of the subshrub $_{2}\left(\frac{1}{3} \cdot \frac{1}{5}\right)$, see Fig. 5 , is the Misiurewicz point $\mathrm{M}_{15,1}$. Note that the period of the representative of branch $\overline{1} \rightarrow 11$ is 14 and, again, the preperiod of a node equals the period of the representative plus one.

A main node of the second subshrub can be calculated by Eq. (16) when $\Sigma_{2}=0$. Then, Eq. (16) becomes Eq. (15) which gives the corresponding ptip of the first subshrub. Therefore, a ptip of the first subshrub is a main node of a portion of the second subshrub.

Let us see now the tips of the second subshrub. We begin by the normal case, when $\Sigma_{2}=0 \cup d_{2 m_{2}}>$ $d_{2\left(m_{2}+1\right)}$. In accordance with our experimental data, a tip $d_{11} d_{12} \cdots d_{1 m_{1}} \overline{d_{1\left(m_{1}+1\right)}} \rightarrow d_{21} d_{22} \cdots d_{2 m_{2}} \overline{d_{2\left(m_{2}+1\right)}}$ of the subshrub $\left(\frac{q_{1}}{p_{1}} \cdot \frac{q_{2}}{p_{2}}\right)$ is a Misiurewicz point $\mathrm{M}_{n^{*}, p^{*}}$ with

$$
\begin{aligned}
& n^{*}=p_{1}\left(p_{2}+\Sigma_{1}-d_{1\left(m_{1}+1\right)}\right)+\Sigma_{2}-d_{2\left(m_{2}+1\right)}+1, \\
& p^{*}=d_{2\left(m_{2}+1\right)},
\end{aligned}
$$

where $\Sigma_{1}=d_{11}+d_{12}+\cdots+d_{1 m_{1}}$ and $\Sigma_{2}=d_{21}+d_{22}+\cdots+d_{2 m_{2}}$. The Eq. (17) is in a non simplified form, in order to better see the final result. However, since we are in the second subshrub, we can simplify Eq. (17) with $d_{1\left(m_{1}+1\right)}=1$. Let us see some explanatory examples in Fig. 5 . In the portion $\overline{1} \rightarrow$, the tip of the path $1,11, \cdots, \overline{1} \rightarrow 2,21, \cdots, 2 \overline{1}$ is the Misiurewicz point $\mathrm{M}_{14,1}$. In the portion $3 \overline{1} \rightarrow$, the tip of the path $3,31, \cdots, 3 \overline{1} \rightarrow 1,11, \cdots, \overline{1}$ is $M_{21,1}$. In the portion $4 \overline{1} \rightarrow$, the tip of the path $4,41, \cdots, 4 \overline{1} \rightarrow$ $1,12,121, \cdots, 12 \overline{1}$ is $\mathrm{M}_{27,1}$.

When $\Sigma_{2} \neq 0 \cap d_{2 m_{2}}<d_{2\left(m_{2}+1\right)}$ we are in the especial case and Eq. (17) becomes

$$
\begin{aligned}
& n^{*}=p_{1}\left(p_{2}+\Sigma_{1}-d_{1\left(m_{1}+1\right)}\right)+\Sigma_{2}-d_{2 m_{2}}+1, \\
& p^{*}=d_{2\left(m_{2}+1\right)} .
\end{aligned}
$$


Equations (17) and (18) only differ in that $\Sigma_{2}-d_{2\left(m_{2}+1\right)}$ changes to $\Sigma_{2}-d_{2 m_{2}}$. Thus, the tip of the path $\overline{1} \rightarrow 1 \overline{2}$ in subshrub $2\left(\frac{1}{3} \cdot \frac{1}{5}\right)$, depicted in Fig. 5, is $\mathrm{M}_{13,2}$.

If we follow the p.u.p. $1,11, \cdots, \overline{1} \rightarrow 1,11, \cdots, \overline{1}$ we reach the more prominent tip of $\operatorname{shrub}\left(\frac{q_{1}}{p_{1}} \cdot \frac{q_{2}}{p_{2}}\right)$, that we name ftip $\left(\frac{q_{1}}{p_{1}} \cdot \frac{q_{2}}{p_{2}}\right)$. Now $\Sigma_{1}=\Sigma_{2}=0, d_{2 m_{2}}=0, d_{1\left(m_{1}+1\right)}=d_{2\left(m_{2}+1\right)}=1$, and Eq. (17) becomes in a much more simplified one which give the ftip of a secondary hyperbolic component

$$
\operatorname{ftip}\left(\frac{q_{1}}{p_{1}} \cdot \frac{q_{2}}{p_{2}}\right)=\mathrm{M}_{p_{1}\left(p_{2}-1\right), 1} \text {. }
$$

Indeed, ftip $\left(\frac{1}{3} \cdot \frac{1}{5}\right)=\mathrm{M}_{12,1}$ as can be seen in Fig. 5 .

In this section we have studied the secondary shrubs which are associated to two decorations. Next we shall study the tertiary and $N$-ary shrubs. As we shall see next, the tertiary shrubs are associated to three decorations, the first one a node with $p_{3}$ branches, the second one a node with $p_{2}$ branches and the third one a node with $p_{1}$ branches. That is why we divide the tertiary shrubs into three subshrubs, each one of them associated to one of these decorations. Likewise, the $N$-ary shrubs are associated to $N$ decorations and we divide them into $N$ subshrubs. Let us see them.

\section{Tertiary Shrubs}

We denominate tertiary hyperbolic component $\frac{q_{1}}{p_{1}} \cdot \frac{q_{2}}{p_{2}} \cdot \frac{q_{3}}{p_{3}}$ the hyperbolic component attached to the secondary hyperbolic component $\frac{q_{1}}{p_{1}} \cdot \frac{q_{2}}{p_{2}}$, in the relative position that $\frac{q_{3}}{p_{3}}$ should have with regard to the main cardioid. From its $\operatorname{MF}\left(\frac{q_{1}}{p_{1}} \cdot \frac{q_{2}}{p_{2}} \cdot \frac{q_{3}}{p_{3}}\right)$ point a tertiary $\operatorname{shrub}\left(\frac{q_{1}}{p_{1}} \cdot \frac{q_{2}}{p_{2}} \cdot \frac{q_{3}}{p_{3}}\right)$ emerges formed by $\operatorname{subshrub}_{1}\left(\frac{q_{1}}{p_{1}} \cdot \frac{q_{2}}{p_{2}} \cdot \frac{q_{3}}{p_{3}}\right)$, subshrub $2\left(\frac{q_{1}}{p_{1}} \cdot \frac{q_{2}}{p_{2}} \cdot \frac{q_{3}}{p_{3}}\right)$ and $\operatorname{subshrub}_{3}\left(\frac{q_{1}}{p_{1}} \cdot \frac{q_{2}}{p_{2}} \cdot \frac{q_{3}}{p_{3}}\right)$.

Figure 6 shows the tertiary shrub $\left(\frac{1}{3} \cdot \frac{1}{4} \cdot \frac{1}{5}\right)$, and Fig. 7 shows a sketch of this shrub. From each node of subshrub $_{1}\left(\frac{1}{3} \cdot \frac{1}{4} \cdot \frac{1}{5}\right)$, which is born from the $\operatorname{MF}\left(\frac{1}{3} \cdot \frac{1}{4} \cdot \frac{1}{5}\right)$, five branches emanate; from the nodes of the $\operatorname{subshrub}_{2}\left(\frac{1}{3} \cdot \frac{1}{4} \cdot \frac{1}{5}\right)$, which are born from the ptips of $\operatorname{subshrub}_{1}\left(\frac{1}{3} \cdot \frac{1}{4} \cdot \frac{1}{5}\right)$, four branches emanate; and from the nodes of the $\operatorname{subshrub}_{3}\left(\frac{1}{3} \cdot \frac{1}{4} \cdot \frac{1}{5}\right)$, which are born from the ptips of $\operatorname{subshrub}_{2}\left(\frac{1}{3} \cdot \frac{1}{4} \cdot \frac{1}{5}\right)$, three branches emanate.

A tertiary shrub is yet more complex than a secondary shrub, therefore, let us analyse it carefully by using Fig. 7. Again, it is not easy to see the correspondence between the shrub $\left(\frac{1}{3} \cdot \frac{1}{4} \cdot \frac{1}{5}\right)$ of Fig. 6 and its sketch of Fig. 7. As in primary and secondary shrubs, we can reach a tip of the first subshrub by following an u.p. $d_{11}, d_{11} d_{12}, \cdots, d_{11} d_{12} \cdots d_{1 m_{1}} \overline{d_{1\left(m_{1}+1\right)}}$ with a first step $d_{11}, d_{11} d_{12}, \cdots, d_{11} d_{12} \cdots d_{1 m_{1}}$ until a level $m_{1}$ representative $p_{r_{1}}$ (that can exist or not) and a second step $\overline{d_{1\left(m_{1}+1\right)}}$ to reach the tip. If $d_{1\left(m_{1}+1\right)} \neq 1$ we reach a crisis point tip and the second subshrub is not reached, but if $d_{1\left(m_{1}+1\right)}=1$ we reach a portion of the second subshrub. There is an infinity of paths to reach the second subshrub, and therefore there is an infinity of non-connected separated portions, each one emerging from a ptip of the first subshrub. We have marked with a little black circle the ends of the p.u.p. and with a little black square the ends of the other u.p. in Fig. 7, where we show

four portions of the second subshrub, $\overline{1} \rightarrow, 2 \overline{1} \rightarrow, 3 \overline{1} \rightarrow$ and $4 \overline{1} \rightarrow$, that are born in the main nodes $\mathrm{M}_{49,3}, \mathrm{M}_{73,3}, \mathrm{M}_{85,3}$ and $\mathrm{M}_{97,3}$. Likewise, starting from a ptip of the first subshrub we can reach a tip of the second subshrub by following an u.p. $d_{21}, d_{21} d_{22}, \cdots, d_{21} d_{22} \cdots d_{2 m_{2}} \overline{d_{2\left(m_{2}+1\right)}}$ in each one of the infinite

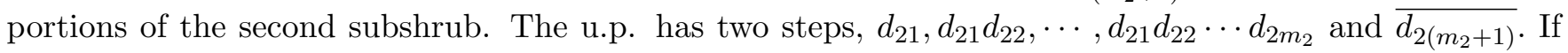
$d_{2\left(m_{2}+1\right)} \neq 1$ we reach a crisis point tip, but if $d_{2\left(m_{2}+1\right)}=1$ we reach a ptip point and a portion of the third subshrub is reached. The third subshrub has an infinity of non-connected portions, each one emerging from a ptip of any of the infinite portions of the second subshrub. Figure 7 shows two portions of the third subshrub starting from any of the four portions of the second subshrub (eight portions in total). These portions are $\overline{1} \rightarrow \overline{1} \rightarrow\left(\right.$ born in $\left.M_{46,1}\right), \overline{1} \rightarrow 2 \overline{1} \rightarrow\left(\right.$ born in $\left.M_{52,1}\right), 2 \overline{1} \rightarrow \overline{1} \rightarrow\left(\right.$ born in $\left.M_{70,1}\right), 2 \overline{1} \rightarrow 2 \overline{1} \rightarrow($ born in $\left.\mathrm{M}_{76,1}\right), 3 \overline{1} \rightarrow \overline{1} \rightarrow\left(\right.$ born in $\left.\mathrm{M}_{82,1}\right), 3 \overline{1} \rightarrow 3 \overline{1} \rightarrow\left(\right.$ born in $\left.\mathrm{M}_{91,1}\right), 4 \overline{1} \rightarrow \overline{1} \rightarrow\left(\right.$ born in $\left.\mathrm{M}_{94,1}\right)$ and $4 \overline{1} \rightarrow 3 \overline{1} \rightarrow$ 


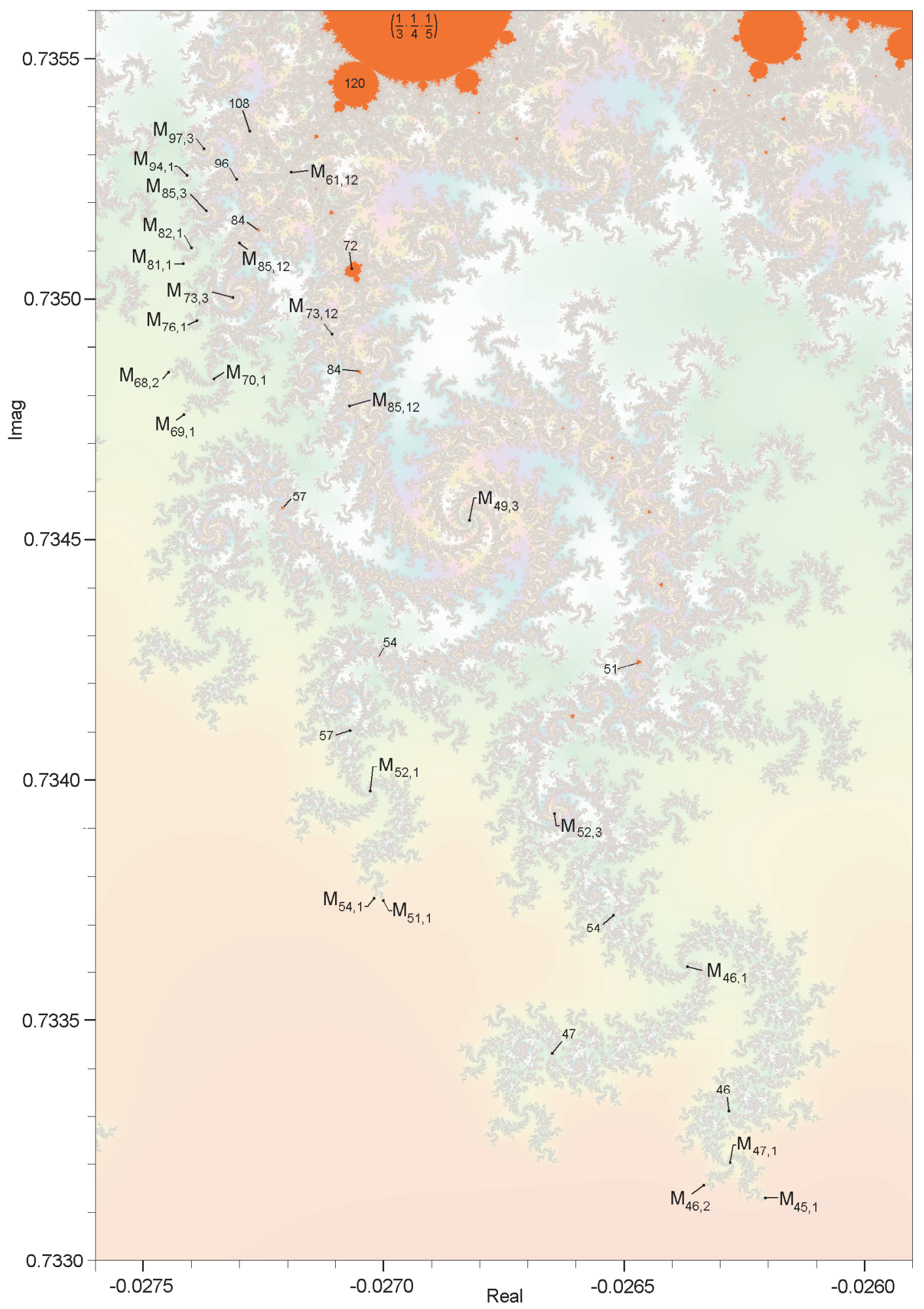

Figure 6: Mandelbrot set region showing a tertiary shrub, shrub $\left(\frac{1}{3} \cdot \frac{1}{4} \cdot \frac{1}{5}\right)$.

(born in $\mathrm{M}_{103,1}$ ). All these Misiurewicz points are ptips of the second subshrub and therefore main nodes of the third subshrub.

Likewise, starting from a ptip of the second subshrub we can reach a tip of the third subshrub by 


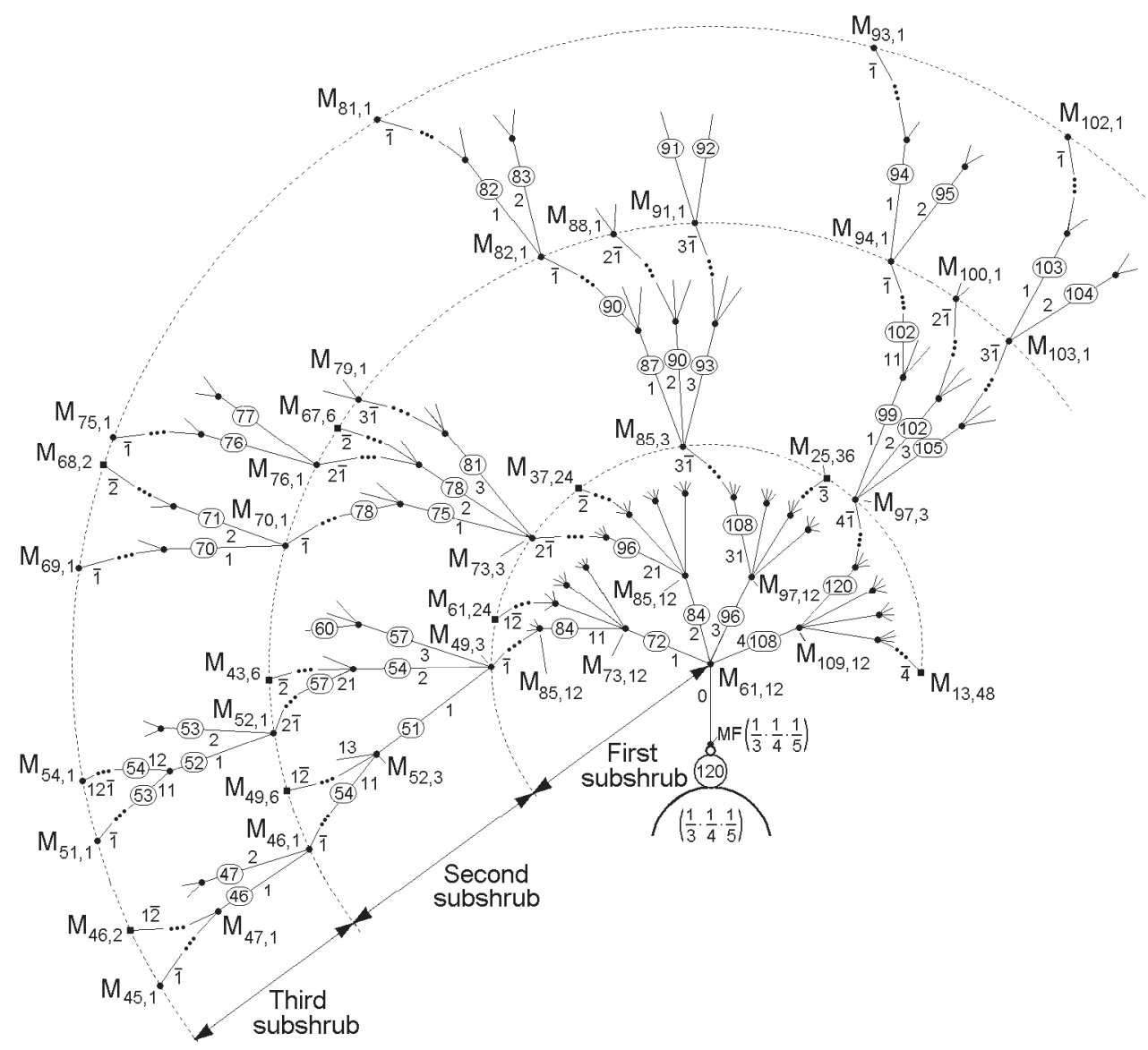

Figure 7: Sketch of the tertiary shrub of Fig. 6, shrub $\left(\frac{1}{3} \cdot \frac{1}{4} \cdot \frac{1}{5}\right)$, and its three subshrubs. The second and third subshrubs have an infinity of portions. Four of these portions of the second subshrub are shown (those coming from $\overline{1}, 2 \overline{1}, 3 \overline{1}$ and $4 \overline{1}$ of the first subshrub). Two portions starting from each one of the four portions of the second subshrub (eight portions in total) are shown in the third subshrub.

following an u.p. $d_{31}, d_{31} d_{32}, \cdots, d_{31} d_{32} \cdots d_{3 m_{3}} \overline{d_{3\left(m_{3}+1\right)}}$ in each one of the infinite portions of the third subshrub. Now, since we are in the last subshrub, either $d_{3\left(m_{3}+1\right)} \neq 1$ or $d_{3\left(m_{3}+1\right)}=1$ we reach a tip which always is a crisis point.

\subsection{First subshrub hyperbolic components ordering}

In accordance with the experimental data, the period of the representative of the branch $d_{11} d_{12} \ldots d_{1 m_{1}}$ of the subshrub $1\left(\frac{q_{1}}{p_{1}} \cdot \frac{q_{2}}{p_{2}} \cdot \frac{q_{3}}{p_{3}}\right)$ is

$$
p_{r_{1}}=p_{1} p_{2}\left(p_{3}+\Sigma_{1}\right) \text {, }
$$

where $\Sigma_{1}=d_{11}+d_{12}+\cdots+d_{1 m_{1}}$. Thus, the representative of branch 21 of subshrub $1\left(\frac{1}{3} \cdot \frac{1}{4} \cdot \frac{1}{5}\right)$ has period 96, and the representative of branch 41 has period 120 (Fig. 7).

\subsection{Second subshrub hyperbolic components ordering}

To calculate the period of any level $m_{2}$ branch representative of a subshrub $2\left(\frac{q_{1}}{p_{1}} \cdot \frac{q_{2}}{p_{2}} \cdot \frac{q_{3}}{p_{3}}\right)$ portion, we have to know the complete associated number of the branch $d_{11} d_{12} \cdots d_{1 m_{1}} \overline{d_{1\left(m_{1}+1\right)}} \rightarrow d_{21} d_{22} \cdots d_{2 m_{2}}$, with 
$d_{1\left(m_{1}+1\right)}=1$. The period is

$$
p_{r_{2}}=p_{1}\left[p_{2}\left(p_{3}+\Sigma_{1}-1\right)+\Sigma_{2}\right]
$$

where $\Sigma_{1}=d_{11}+d_{12}+\cdots+d_{1 m_{1}}$ (characteristic of the portion where the branch is) and $\Sigma_{2}=d_{21}+$ $d_{22}+\cdots+d_{2 m_{2}}$ (characteristic of the branch). Thus, the periods of representatives of branches $\overline{1} \rightarrow 21$ and $4 \overline{1} \rightarrow 11$ of $\operatorname{subshrub}_{2}\left(\frac{1}{3} \cdot \frac{1}{4} \cdot \frac{1}{5}\right)$ are 57 and 102 (Fig. 7).

\subsection{Third subshrub hyperbolic component ordering}

If we know the complete associated number of the branch, $d_{11} d_{12} \cdots d_{1 m_{1}} \overline{d_{1\left(m_{1}+1\right)}} \rightarrow$ $d_{21} d_{22} \cdots d_{2 m_{2}} \overline{d_{2\left(m_{2}+1\right)}} \rightarrow d_{31} d_{32} \cdots d_{3 m_{3}}$, where $d_{1\left(m_{1}+1\right)}=d_{2\left(m_{2}+1\right)}=1$, the period of any level $m_{3}$ branch representative of a subshrub $3\left(\frac{q_{1}}{p_{1}} \cdot \frac{q_{2}}{p_{2}} \cdot \frac{q_{3}}{p_{3}}\right)$ portion is

$$
p_{r_{3}}=p_{1}\left[p_{2}\left(p_{3}+\Sigma_{1}-1\right)+\Sigma_{2}-1\right]+\Sigma_{3},
$$

where $\Sigma_{1}=d_{11}+d_{12}+\cdots+d_{1 m_{1}}$ and $\Sigma_{2}=d_{21}+d_{22}+\cdots+d_{2 m_{2}}$ are characteristic of the portion where the branch is, and $\Sigma_{3}=d_{31}+d_{32}+\cdots+d_{3 m_{3}}$ is characteristic of the branch. Thus, the periods of representatives of branches $\overline{1} \rightarrow 2 \overline{1} \rightarrow 12$ and $4 \overline{1} \rightarrow 3 \overline{1} \rightarrow 2$ of $\operatorname{subshrub}_{3}\left(\frac{1}{3} \cdot \frac{1}{4} \cdot \frac{1}{5}\right)$ are 54 and 104 (Fig. 7).

Let us study the Misiurewicz points of the nodes and tips of the three subshrubs of the tertiary $\operatorname{shrub}\left(\frac{1}{3} \cdot \frac{1}{4} \cdot \frac{1}{5}\right)$ (see Fig. 7 ). We begin with the first subshrub.

\subsection{First subshrub Misiurewicz points ordering}

A level $m_{1}$ node $d_{11} d_{12} \cdots d_{1 m_{1}}$ of the subshrub $\left(\frac{q_{1}}{p_{1}} \cdot \frac{q_{2}}{p_{2}} \cdot \frac{q_{3}}{p_{3}}\right)$ is a non-characteristic Misiurewicz point $\mathrm{M}_{n^{*}, p^{*}}$ with

$$
n^{*}=p_{1} p_{2}\left(p_{3}+\Sigma_{1}\right)+1, p^{*}=p_{1} p_{2},
$$

where $\Sigma_{1}=d_{11}+d_{12}+\cdots+d_{1 m_{1}}$. Thus, the nodes $1,2,3$ and 4 of subshrub $1\left(\frac{1}{3} \cdot \frac{1}{4} \cdot \frac{1}{5}\right)$ in Fig. 7 are the Misiurewicz points $\mathrm{M}_{73,12}, \mathrm{M}_{85,12}, \mathrm{M}_{97,12}$ and $\mathrm{M}_{109,12}$.

Let us see now tips. To reach a tip we have to follow an u.p. $d_{11}, d_{11} d_{12}, \cdots, d_{11} d_{12} \cdots d_{1 m_{1}} \overline{d_{1\left(m_{1}+1\right)}}$ with two steps. We have again a normal case and an especial case. As can experimentally be seen, in the normal case, $\Sigma_{1}=0 \cup d_{1 m_{1}}>d_{1\left(m_{1}+1\right)}$, the tip is the Misiurewicz point $\mathrm{M}_{n^{*}, p^{*}}$ with

$$
\begin{aligned}
& n^{*}=p_{1} p_{2}\left(p_{3}+\Sigma_{1}-d_{1\left(m_{1}+1\right)}\right)+1, \\
& p^{*}=p_{1} p_{2} d_{1\left(m_{1}+1\right)},
\end{aligned}
$$

where $\Sigma_{1}=d_{11}+d_{12}+\cdots+d_{1 m_{1}}$. For example, if we follow the paths $2,22, \cdots, \overline{2} ; 3,33, \cdots, \overline{3}$ and $4,44, \cdots, \overline{4}$ of subshrub $1\left(\frac{1}{3} \cdot \frac{1}{4} \cdot \frac{1}{5}\right)$ in Fig. 7 we obtain $\mathrm{M}_{37,24}, \mathrm{M}_{25,36}$ and $\mathrm{M}_{13,48}$.

When $\Sigma_{1} \neq 0 \cap d_{1 m_{1}}<d_{1\left(m_{1}+1\right)}$ we are in the especial case and Eq. (24) becomes

$$
\begin{aligned}
& n^{*}=p_{1} p_{2}\left(p_{3}+\Sigma_{1}-d_{1 m_{1}}\right)+1, \\
& p^{*}=p_{1} p_{2} d_{1\left(m_{1}+1\right)} .
\end{aligned}
$$

Equations (24) and (25) only differ in that $\Sigma_{1}-d_{1\left(m_{1}+1\right)}$ changes to $\Sigma_{1}-d_{1 m_{1}}$. Thus, if we follow the path $1,12, \cdots, 1 \overline{2}$ of $\operatorname{subshrub}_{1}\left(\frac{1}{3} \cdot \frac{1}{4} \cdot \frac{1}{5}\right)$ in Fig. 7 we obtain $\mathrm{M}_{61,24}$.

Again, if we follow a p.u.p., $d_{1\left(m_{1}+1\right)}=1$, we reach a main node of the second subshrub. We are in the normal case, and therefore it seems we should apply Eq. (24). However, in this case, since we are not in the first subshrub but in the second one, the period of the Misiurewicz point is $p_{1}$ as we shall see in the next section. Hence, Eq. (24) becomes

$$
n^{*}=p_{1} p_{2}\left(p_{3}+\Sigma_{1}-1\right)+1, p^{*}=p_{1} .
$$

Thus, if we follow the p.u.p. $1,11, \cdots, \overline{1}$ and $4,41, \cdots, 4 \overline{1}$ of $\operatorname{subshrub}_{1}\left(\frac{1}{3} \cdot \frac{1}{4} \cdot \frac{1}{5}\right)$ in Fig. 7 , we reach the ptips $\mathrm{M}_{49,3}$ and $\mathrm{M}_{97,3}$. 


\subsection{Second subshrub Misiurewicz points ordering}

A portion of the subshrub $2\left(\frac{1}{3} \cdot \frac{1}{4} \cdot \frac{1}{5}\right)$ emanates from any ptip of the first subshrub. We can see experimentally that a level $m_{2}$ node $d_{11} d_{12} \cdots d_{1 m_{1}} \overline{d_{1\left(m_{1}+1\right)}} \rightarrow d_{21} d_{22} \cdots d_{2 m_{2}}$ of the subshrub $2\left(\frac{q_{1}}{p_{1}} \cdot \frac{q_{2}}{p_{2}} \cdot \frac{q_{3}}{p_{3}}\right)$ is a non-characteristic Misiurewicz point $\mathrm{M}_{n^{*}, p^{*}}$ with

$$
n^{*}=p_{1}\left[p_{2}\left(p_{3}+\Sigma_{1}-1\right)+\Sigma_{2}\right]+1, p^{*}=p_{1},
$$

where $\Sigma_{1}=d_{11}+d_{12}+\cdots+d_{1 m_{1}}$ and $\Sigma_{2}=d_{21}+d_{22}+\cdots+d_{2 m_{2}}$. Let us note that a ptip of the first subshrub, Eq. (26), is a main node of the second subshrub, Eq. (27), when $\Sigma_{2}=0$.

Let us see now tips. As we can see in Fig. 7, in the normal case $\Sigma_{2}=0 \cup d_{2 m_{2}}>d_{2\left(m_{2}+1\right)}$ the tip is the Misiurewicz point $\mathrm{M}_{n^{*}, p^{*}}$ with

$$
\begin{aligned}
& n^{*}=p_{1}\left[p_{2}\left(p_{3}+\Sigma_{1}-d_{1\left(m_{1}+1\right)}\right)+\Sigma_{2}-d_{2\left(m_{2}+1\right)}\right]+1, \\
& p^{*}=p_{1} d_{2\left(m_{2}+1\right)}
\end{aligned}
$$

(we prefer to give the non-simplified form of Eq. (28) even though we know that $d_{1\left(m_{1}+1\right)}=1$ if we are in the second subshrub). For example, if we follow the complete paths $1,11, \cdots, \overline{1} \rightarrow 2,22, \cdots, \overline{2}$ and $2,21, \cdots, 2 \overline{1} \rightarrow 2,22, \cdots, \overline{2}$ of $\operatorname{subshrub}_{1}\left(\frac{1}{3} \cdot \frac{1}{4} \cdot \frac{1}{5}\right)$ in Fig. 7 we obtain the crisis points $\mathrm{M}_{43,6}$ and $\mathrm{M}_{67,6}$.

When $\Sigma_{2} \neq 0 \cap d_{2 m_{2}}<d_{2(m+1)}$ we are in the especial case and Eq. (28) becomes

$$
\begin{aligned}
& n^{*}=p_{1}\left[p_{2}\left(p_{3}+\Sigma_{1}-d_{1\left(m_{1}+1\right)}\right)+\Sigma_{2}-d_{2 m_{2}}\right]+1, \\
& p^{*}=p_{1} d_{2\left(m_{2}+1\right)} .
\end{aligned}
$$

Equations (28) and (29) only differ in that $\Sigma_{2}-d_{2\left(m_{2}+1\right)}$ changes to $\Sigma_{2}-d_{2 m_{2}}$. For example, if we follow the complete path $1,11, \cdots, \overline{1} \rightarrow 1,12, \cdots, 1 \overline{2}$ of $\operatorname{subshrub}_{1}\left(\frac{1}{3} \cdot \frac{1}{4} \cdot \frac{1}{5}\right)$ in Fig. 7 we obtain the crisis point $\mathrm{M}_{49,6}$.

If we follow a p.u.p., $d_{2\left(m_{2}+1\right)}=1$, we reach a main node of the third subshrub. We are in the normal case, and therefore it seems we should apply Eq. (28). However, in this case, since we are not in the second subshrub but in the third one, the last one, the period of the Misiurewicz point must be one. Hence, Eq. (28) becomes

$$
\begin{aligned}
& n^{*}=p_{1}\left[p_{2}\left(p_{3}+\Sigma_{1}-d_{1\left(m_{1}+1\right)}\right)+\Sigma_{2}-1\right]+1, \\
& p^{*}=1 .
\end{aligned}
$$

Thus, if we follow the path $1,11, \cdots, \overline{1} \rightarrow 2,21, \cdots, 2 \overline{1}$ of $\operatorname{subshrub}_{1}\left(\frac{1}{3} \cdot \frac{1}{4} \cdot \frac{1}{5}\right)$ in Fig. 7 we reach the ptip $\mathrm{M}_{52,1}$.

\subsection{Third subshrub Misiurewicz points ordering}

A portion of $\operatorname{subshrub}_{3}\left(\frac{q_{1}}{p_{1}} \cdot \frac{q_{2}}{p_{2}} \cdot \frac{q_{3}}{p_{3}}\right)$ emanates from any ptip of any second subshrub portion. A level $m_{3}$ node $d_{11} d_{12} \cdots d_{1 m_{1}} \overline{d_{1\left(m_{1}+1\right)}} \rightarrow d_{21} d_{22} \cdots d_{2 m_{2}} \overline{d_{2\left(m_{2}+1\right)}} \rightarrow d_{31} d_{32} \cdots d_{3 m_{3}}$ of $\operatorname{subshrub}_{3}\left(\frac{q_{1}}{p_{1}} \cdot \frac{q_{2}}{p_{2}} \cdot \frac{q_{3}}{p_{3}}\right)$ is a characteristic Misiurewicz point $\mathrm{M}_{n^{*}, p^{*}}$ with

$$
\begin{aligned}
& n^{*}=p_{1}\left[p_{2}\left(p_{3}+\Sigma_{1}-1\right)+\Sigma_{2}-1\right]+\Sigma_{3}+1, \\
& p^{*}=1 .
\end{aligned}
$$

Let us note again that a ptip of the second subshrub, Eq. (30), is a main node of the third subshrub, Eq. (31), when $\Sigma_{3}=0$. 
Let us see now tips. If we are in the normal case, $\Sigma_{3}=0 \cup d_{3 m_{3}}>d_{3\left(m_{3}+1\right)}$, the tip is the Misiurewicz point $\mathrm{M}_{n^{*}, p^{*}}$ with

$$
\begin{aligned}
& n^{*}=p_{1}\left[p_{2}\left(p_{3}+\Sigma_{1}-d_{1\left(m_{1}+1\right)}\right)+\Sigma_{2}-d_{2\left(m_{2}+1\right)}\right] \\
& +\Sigma_{3}-d_{3\left(m_{3}+1\right)}+1, p^{*}=d_{3\left(m_{3}+1\right)} .
\end{aligned}
$$

Note that we give Eq. (32) in a non-simplified form. Thus, if we follow the complete path $2,21, \cdots, 2 \overline{1} \rightarrow$ $1,11, \cdots, \overline{1} \rightarrow 2,22, \cdots, \overline{2}$ of $\operatorname{shrub}\left(\frac{1}{3} \cdot \frac{1}{4} \cdot \frac{1}{5}\right)$ in Fig. 7 we obtain $\mathrm{M}_{68,2}$.

When $\Sigma_{3} \neq 0 \cap d_{3 m}<d_{3(m+1)}$ we are in the especial case and Eq. (32) becomes

$$
\begin{aligned}
& n^{*}=p_{1}\left[p_{2}\left(p_{3}+\Sigma_{1}-d_{1\left(m_{1}+1\right)}\right)+\Sigma_{2}-d_{2\left(m_{2}+1\right)}\right] \\
& +\Sigma_{3}-d_{3 m_{3}}+1, p^{*}=d_{3\left(m_{3}+1\right)} .
\end{aligned}
$$

Equations (32) and (33) only differ in that $\Sigma_{3}-d_{3\left(m_{3}+1\right)}$ changes to $\Sigma_{3}-d_{3 m_{3}}$. For example, see Fig. 7, if we follow the complete path $1,11, \cdots, \overline{1} \rightarrow 1,11, \cdots, \overline{1} \rightarrow 1,12, \cdots, 1 \overline{2}$ we obtain $\mathrm{M}_{46,2}$.

Since the third subshrub is the last one, its tips are always crisis points. If we follow the p.u.p. $1,11, \cdots, \overline{1}$ in the first, second and third subshrubs of the shrub $\left(\frac{q_{1}}{p_{1}} \cdot \frac{q_{2}}{p_{2}} \cdot \frac{q_{3}}{p_{3}}\right)$ we reach the more prominent tip, that we shall call ftip $\left(\frac{q_{1}}{p_{1}} \cdot \frac{q_{2}}{p_{2}} \cdot \frac{q_{3}}{p_{3}}\right)$. Now $\Sigma_{1}=\Sigma_{2}=\Sigma_{3}=0$, and $d_{1\left(m_{1}+1\right)}=d_{2\left(m_{2}+1\right)}=d_{3\left(m_{3}+1\right)}=$ 1. Hence, Eq. (32) becomes in a much more simplified one which give the ftip of a tertiary hyperbolic component:

$$
\operatorname{ftip}\left(\frac{q_{1}}{p_{1}} \cdot \frac{q_{2}}{p_{2}} \cdot \frac{q_{3}}{p_{3}}\right)=\mathrm{M}_{p_{1}\left[p_{2}\left(p_{3}-1\right)-1\right], 1} .
$$

Thus, as we can see in Figs. 6 and 7,

$$
\operatorname{ftip}\left(\frac{1}{3} \cdot \frac{1}{4} \cdot \frac{1}{5}\right)=\mathrm{M}_{45,1}
$$

\section{$5 \quad$ Quinary Shrubs}

Next, let us see the case of a quinary shrub, the $\operatorname{shrub}\left(\frac{1}{3} \cdot \frac{1}{2} \cdot \frac{1}{2} \cdot \frac{1}{2} \cdot \frac{1}{3}\right)$ shown in Fig. 8(a). Two enlargements of this figure are shown in Fig. 8(b) and 8(c). Obviously, a quinary shrub is yet much more complex than a tertiary one and, therefore, let us analyze it carefully by using Fig. 9 where a sketch of $\operatorname{shrub}\left(\frac{1}{3} \cdot \frac{1}{2} \cdot \frac{1}{2} \cdot \frac{1}{2} \cdot \frac{1}{3}\right)$ is depicted with its five subshrubs clearly separated. From each node of the subshrub $1\left(\frac{1}{3} \cdot \frac{1}{2} \cdot \frac{1}{2} \cdot \frac{1}{2} \cdot \frac{1}{3}\right)$ three branches emanate, from the nodes of the second, third and fourth subshrubs two branches emanate, and from the nodes of the fifth subshrub three branches emanate. Since from the second, third and fourth subshrubs two branches emanate from each node, these three subshrubs look like a single continuous branch. By following any u.p. of the first subshrub, $d_{11}, d_{11} d_{12}, \cdots, d_{11} d_{12}, \cdots, d_{1 m_{1}} \overline{d_{1\left(m_{1}+1\right)}}$, we can reach a tip, but we only reach the second subshrub if we follow a p.u.p., i.e. if $d_{1\left(m_{1}+1\right)}=1$. This second subshrub has an infinity of non-connected portions, each one emerging from a ptip of the first subshrub. We show three portions of the second subshrub $\overline{1} \rightarrow, 12 \overline{1} \rightarrow$ and $2 \overline{1} \rightarrow$, born in $\mathrm{M}_{49,12}, \mathrm{M}_{121,12}$ and $\mathrm{M}_{97,12}$. The second, third and fourth subshrubs have one only path $1,11, \cdots, \overline{1}$ and therefore from the end of any portion only a new portion can emerge. The three portions of the third subshrub are $\overline{1} \rightarrow \overline{1} \rightarrow, 12 \overline{1} \rightarrow \overline{1}$ and $2 \overline{1} \rightarrow \overline{1} \rightarrow$, born in $\mathrm{M}_{37,6}, \mathrm{M}_{109,6}$ and $\mathrm{M}_{85,6}$. The three portions of the fourth subshrub are $\overline{1} \rightarrow \overline{1} \rightarrow \overline{1} \rightarrow$, $12 \overline{1} \rightarrow \overline{1} \rightarrow \overline{1} \rightarrow$ and $2 \overline{1} \rightarrow \overline{1} \rightarrow \overline{1} \rightarrow$, born in $\mathrm{M}_{31,3}, \mathrm{M}_{103,3}$ and $\mathrm{M}_{79,3}$. Finally, the three portions of the fifth subshrub are $\overline{1} \rightarrow \overline{1} \rightarrow \overline{1} \rightarrow \overline{1} \rightarrow, 12 \overline{1} \rightarrow \overline{1} \rightarrow \overline{1} \rightarrow \overline{1} \rightarrow$ and $2 \overline{1} \rightarrow \overline{1} \rightarrow \overline{1} \rightarrow \overline{1} \rightarrow$, born in $\mathrm{M}_{28,1}, \mathrm{M}_{100,1}$ and $\mathrm{M}_{76,1}$. Likewise, starting from a main node of the fifth subshrub we can reach a tip by following 


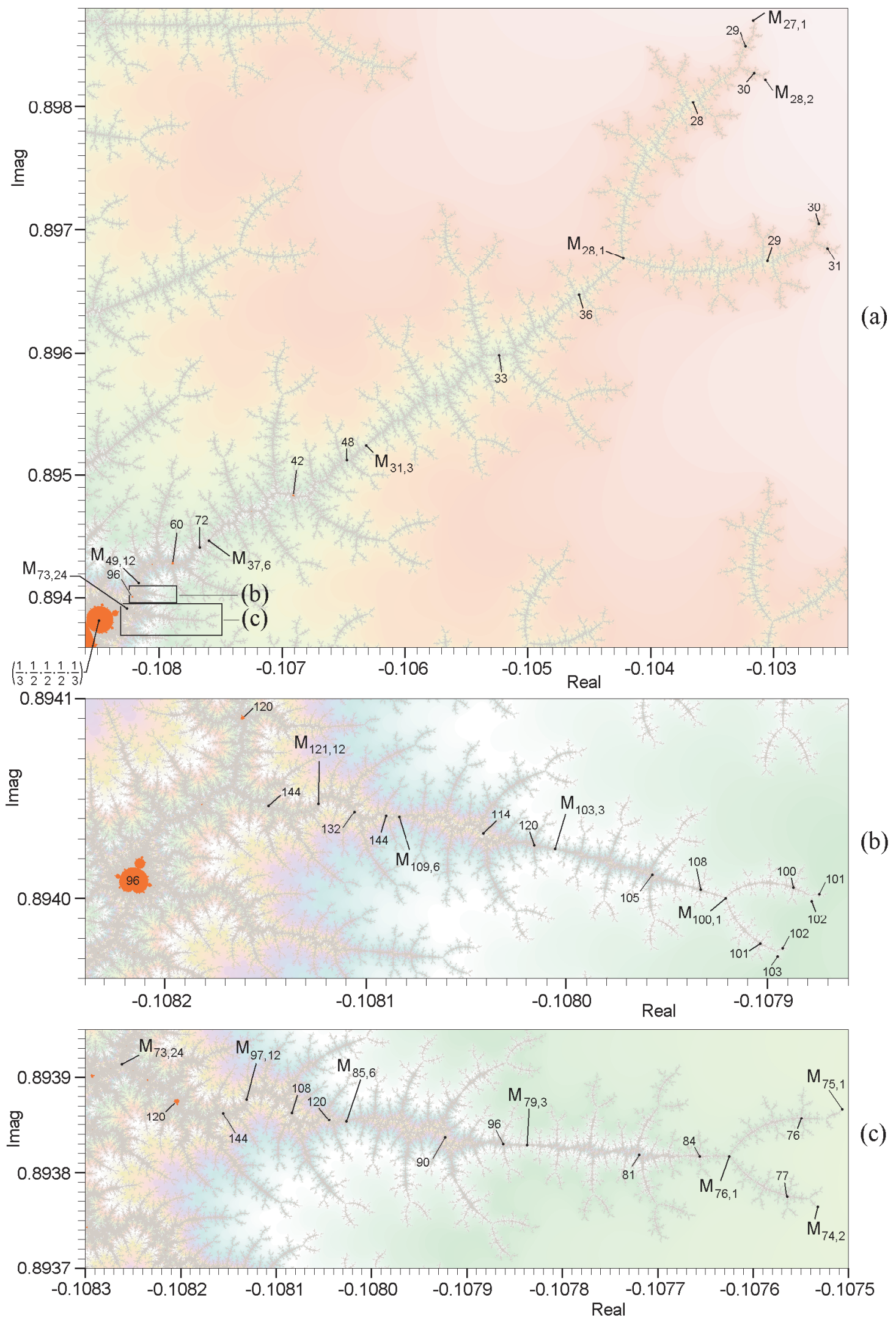

Figure 8: a) Mandelbrot set region showing a quinary shrub, shrub $\left(\frac{1}{3} \cdot \frac{1}{2} \cdot \frac{1}{2} \cdot \frac{1}{2} \cdot \frac{1}{3}\right)$. b) and c) Enlargements of the subregions shown in a). 


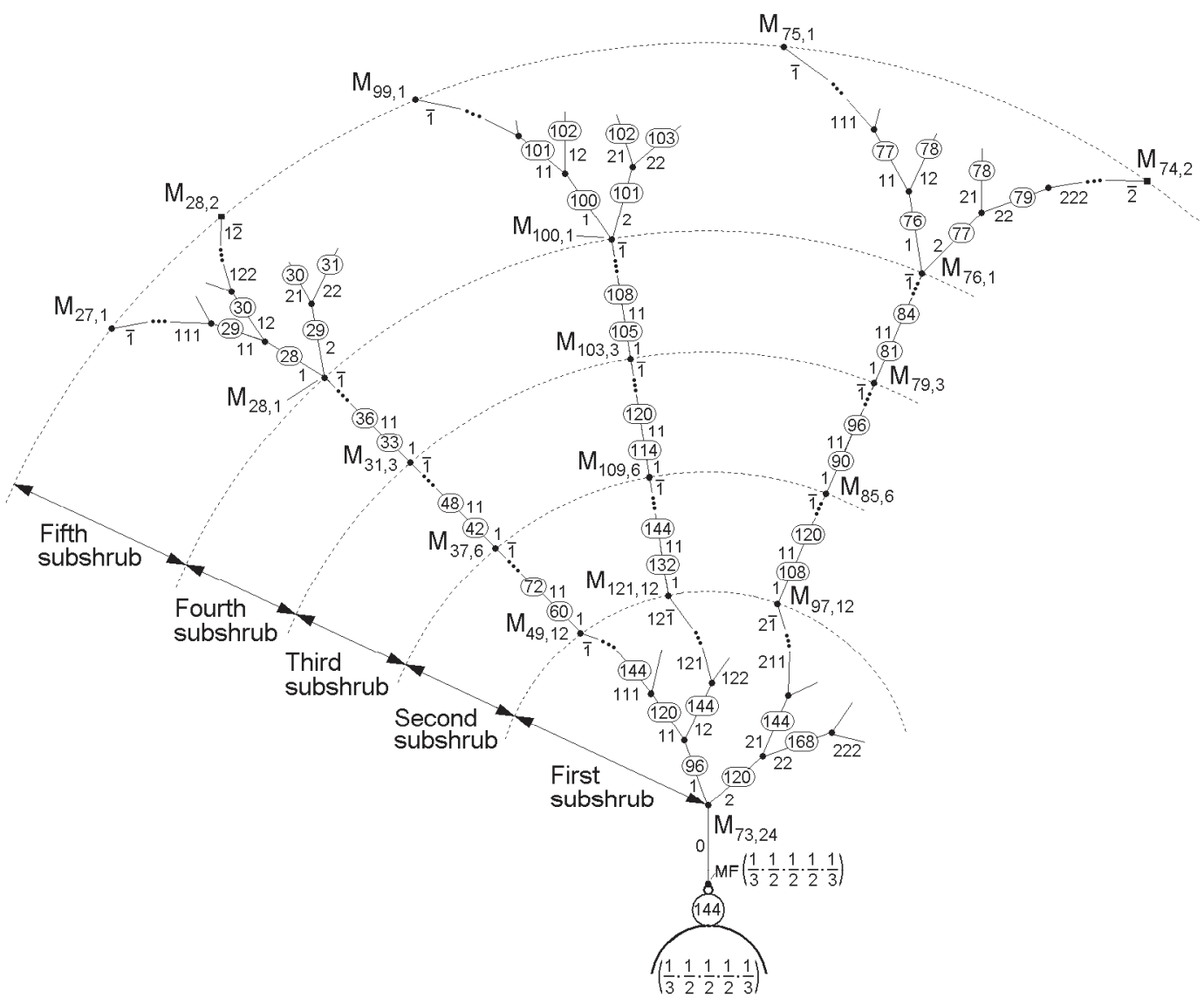

Figure 9: Sketch of the quinary shrub of Fig. $8(\mathrm{a})$, shrub $\left(\frac{1}{3} \cdot \frac{1}{2} \cdot \frac{1}{2} \cdot \frac{1}{2} \cdot \frac{1}{3}\right)$ and its five subshrubs. The second subshrub has an infinity of portions and three of them are depicted on the figure (those coming from $\overline{1}, 12 \overline{1}$ and $2 \overline{1}$ ). The second, third and forth subshrubs are linear, and only a portion come from each portion.

an u.p. $d_{51} d_{52} \cdots d_{5 m_{5}} \overline{d_{5\left(m_{5}+1\right)}}$. Since the fifth subshrub is the last one, both ptips $d_{5\left(m_{5}+1\right)}=1$ and tips $d_{5\left(m_{5}+1\right)} \neq 1$ are always crisis points.

In accordance with the experimental data, the period of the representative of the branch $d_{11} d_{12} \cdots$ $d_{1 m_{1}}$ of the subshrub $1\left(\frac{q_{1}}{p_{1}} \cdot \frac{q_{2}}{p_{2}} \cdot \frac{q_{3}}{p_{3}} \cdot \frac{q_{4}}{p_{4}} \cdot \frac{q_{5}}{p_{5}}\right)$ is

$$
p_{r_{1}}=p_{1} p_{2} p_{3} p_{4}\left(p_{5}+\Sigma_{1}\right)
$$

where $\Sigma_{1}=d_{11}+d_{12}+\cdots+d_{1 m_{1}}$. Thus, the period of the representative of the branch 12 of subshrub $_{1}\left(\frac{1}{3} \cdot \frac{1}{2} \cdot \frac{1}{2} \cdot \frac{1}{2} \cdot \frac{1}{3}\right)$ is 144 , the same of the representative of the branch 21 of the same subshrub (see Fig. 9).

The period of the representative of the branch $d_{11} d_{12} \cdots d_{1 m_{1}} \overline{d_{1\left(m_{1}+1\right)}} \rightarrow d_{21} d_{22} \cdots d_{2 m_{2}}$ of a subshrub $_{2}\left(\frac{q_{1}}{p_{1}} \cdot \frac{q_{2}}{p_{2}} \cdot \frac{q_{3}}{p_{3}} \cdot \frac{q_{4}}{p_{4}} \cdot \frac{q_{5}}{p_{5}}\right)$ portion is

$$
p_{r_{2}}=p_{1} p_{2} p_{3}\left[p_{4}\left(p_{5}+\Sigma_{1}-1\right)+\Sigma_{2}\right] \text {, }
$$

where $\Sigma_{2}=d_{21}+d_{22}+\cdots+d_{2 m_{2}}$. Thus, the period of the representative of the branch $\overline{1} \rightarrow 11$ of $\operatorname{subshrub}_{2}\left(\frac{1}{3} \cdot \frac{1}{2} \cdot \frac{1}{2} \cdot \frac{1}{2} \cdot \frac{1}{3}\right)$ is 72 , and the period of the representative of the branch $12 \overline{1} \rightarrow 1$ of the same subshrub is 132 (see Fig. 9). 
The period of the representative of the branch $d_{11} d_{12} \cdots d_{1 m_{1}} \overline{d_{1\left(m_{1}+1\right)}} \rightarrow d_{21} d_{22} \cdots d_{2 m_{2}} \overline{d_{2\left(m_{2}+1\right)}} \rightarrow$ $d_{31} d_{32} \cdots d_{3 m_{3}}$ of a subshrub $\left(\frac{q_{1}}{p_{1}} \cdot \frac{q_{2}}{p_{2}} \cdot \frac{q_{3}}{p_{3}} \cdot \frac{q_{4}}{p_{4}} \cdot \frac{q_{5}}{p_{5}}\right)$ portion is

$$
\left.p_{r_{3}}=p_{1} p_{2}\left[p_{3}\left[p_{4}\left(p_{5}+\Sigma_{1}-1\right)+\Sigma_{2}-1\right)\right]+\Sigma_{3}\right] \text {, }
$$

where $\Sigma_{3}=d_{31}+d_{32}+\cdots+d_{3 m_{3}}$. Thus, the periods of representatives of branches $\overline{1} \rightarrow \overline{1} \rightarrow 11$ and $12 \overline{1} \rightarrow \overline{1} \rightarrow 11$ of $\operatorname{subshrub}_{3}\left(\frac{1}{3} \cdot \frac{1}{2} \cdot \frac{1}{2} \cdot \frac{1}{2} \cdot \frac{1}{3}\right)$ are 48 and 120 (see Fig. 9).

The period of the representative of the branch $d_{11} d_{12} \cdots d_{1 m_{1}} \overline{d_{1\left(m_{1}+1\right)}} \rightarrow \cdots \rightarrow d_{41} d_{42} \cdots d_{4 m_{4}}$ of a subshrub $_{4}\left(\frac{q_{1}}{p_{1}} \cdot \frac{q_{2}}{p_{2}} \cdot \frac{q_{3}}{p_{3}} \cdot \frac{q_{4}}{p_{4}} \cdot \frac{q_{5}}{p_{5}}\right)$ portion is

$$
p_{r_{4}}=p_{1}\left[p_{2}\left[p_{3}\left[p_{4}\left(p_{5}+\Sigma_{1}-1\right)+\Sigma_{2}-1\right]+\Sigma_{3}-1\right]+\Sigma_{4}\right]
$$

where $\Sigma_{4}=d_{41}+d_{42}+\cdots+d_{4 m_{4}}$. For instance, the periods of representatives of branches $\overline{1} \rightarrow \overline{1} \rightarrow \overline{1} \rightarrow 11$ and $12 \overline{1} \rightarrow \overline{1} \rightarrow \overline{1} \rightarrow 11$ of subshrub $4\left(\frac{q_{1}}{p_{1}} \cdot \frac{q_{2}}{p_{2}} \cdot \frac{q_{3}}{p_{3}} \cdot \frac{q_{4}}{p_{4}} \cdot \frac{q_{5}}{p_{5}}\right)$ are 36 and 108 (see Fig. 9).

The period of the representative of the branch $d_{11} d_{12} \cdots d_{1 m_{1}} \overline{d_{1\left(m_{1}+1\right)}} \rightarrow \cdots \rightarrow d_{51} d_{52} \cdots d_{5 m_{5}}$ of a subshrub $_{5}\left(\frac{q_{1}}{p_{1}} \cdot \frac{q_{2}}{p_{2}} \cdot \frac{q_{3}}{p_{3}} \cdot \frac{q_{4}}{p_{4}} \cdot \frac{q_{5}}{p_{5}}\right)$ portion is

$$
\begin{aligned}
& p_{r_{5}}=p_{1}\left[p_{2}\left[p_{3}\left[p_{4}\left[p_{5}+\Sigma_{1}-1\right)+\Sigma_{2}-1\right]+\Sigma_{3}-1\right]\right. \\
& \left.+\Sigma_{4}-1\right]+\Sigma_{5},
\end{aligned}
$$

where $\Sigma_{5}=d_{51}+d_{52}+\cdots+d_{5 m_{5}}$. For instance, the periods of representatives of branches $\overline{1} \rightarrow \overline{1} \rightarrow \overline{1} \rightarrow$ $\overline{1} \rightarrow 12, \quad 12 \overline{1} \rightarrow \overline{1} \rightarrow \overline{1} \rightarrow \overline{1} \rightarrow 22$ and $2 \overline{1} \rightarrow \overline{1} \rightarrow \overline{1} \rightarrow \overline{1} \rightarrow 21$ are 30, 103 and 78 .

Let us simplify Eqs. (35)-(39). In order to do so, we can name

$$
\begin{aligned}
& p_{1}^{\prime}=p_{1}\left(p_{2}^{\prime}-1\right)+\Sigma_{5}, \\
& p_{2}^{\prime}=p_{2}\left(p_{3}^{\prime}-1\right)+\Sigma_{4}, \\
& p_{3}^{\prime}=p_{3}\left(p_{4}^{\prime}-1\right)+\Sigma_{3}, \\
& p_{4}^{\prime}=p_{4}\left(p_{5}^{\prime}-1\right)+\Sigma_{2}, \\
& p_{5}^{\prime}=p_{5}+\Sigma_{1},
\end{aligned}
$$

where $\Sigma_{1}=d_{11}+d_{12}+\cdots+d_{1 m_{1}}, \ldots$ and $\Sigma_{5}=d_{51}+d_{52}+\cdots+d_{5 m_{5}}$. By taking into account these expressions, Eqs. (35) - (39) can be rewritten in the following simplified form:

$$
\begin{aligned}
& p_{r_{1}}=p_{1} p_{2} p_{3} p_{4} p_{5}^{\prime}, \\
& p_{r_{2}}=p_{1} p_{2} p_{3} p_{4}^{\prime}, \\
& p_{r_{3}}=p_{1} p_{2} p_{3}^{\prime}, \\
& p_{r_{4}}=p_{1} p_{2}^{\prime}, \\
& p_{r_{5}}=p_{1}^{\prime} .
\end{aligned}
$$

If we introduce now the index $i$ that is equal to $1,2, \ldots, 5$ for the first, second, ..., fifth subshrub, we can bring these five formulae in only one:

$$
p_{r_{i}}=p_{1} \cdots p_{5-i} p_{5+1-i}^{\prime}
$$

where $p_{1} \cdots p_{5-i}$ becomes 1 when $i=5$. 


\section{Generalization for N-ary Shrubs}

By generalising, we denominate $N$-ary hyperbolic component $\frac{q_{1}}{p_{1}} \cdot \frac{q_{2}}{p_{2}} \cdots \frac{q_{N}}{p_{N}}$ the hyperbolic component attached to the $(N-1)$-ary hyperbolic component $\frac{q_{1}}{p_{1}} \cdot \frac{q_{2}}{p_{2}} \cdots \frac{q_{N-1}}{p_{N-1}}$, in the relative position that $\frac{q_{N}}{p_{N}}$ should have with regard to the main cardioid. From its Myrberg-Feigenbaum point MF $\left(\frac{q_{1}}{p_{1}} \cdot \frac{q_{2}}{p_{2}} \cdots \frac{q_{N}}{p_{N}}\right)$, a $N$-ary shrub $\left(\frac{q_{1}}{p_{1}} \cdot \frac{q_{2}}{p_{2}} \cdots \frac{q_{N}}{p_{N}}\right)$ emerges that it is formed by $N$ subshrubs, subshrub $1\left(\frac{q_{1}}{p_{1}} \cdot \frac{q_{2}}{p_{2}} \cdots \frac{q_{N}}{p_{N}}\right)$, $\operatorname{subshrub}_{2}\left(\frac{q_{1}}{p_{1}} \cdot \frac{q_{2}}{p_{2}} \cdots \frac{q_{N}}{p_{N}}\right), \cdots, \operatorname{subshrub}_{N}\left(\frac{q_{1}}{p_{1}} \cdot \frac{q_{2}}{p_{2}} \cdots \frac{q_{N}}{p_{N}}\right)$, related to the components $\frac{q_{N}}{p_{N}}, \frac{q_{N-1}}{p_{N-1}} \cdots \frac{q_{1}}{p_{1}}$. From any ptip of the first, second $\cdots(N-1)^{\text {th }}$ subshrub, a second, third $\ldots N^{\text {th }}$ subshrub portion emerges. Likewise, from each node of the first, second $\ldots N^{\text {th }}$ subshrub $p_{N}, p_{N-1} \cdots p_{1}$ branches emanate.

\subsection{Generalization for representatives}

We shall generalize Eqs. (35) to (39) for shrub $\left(\frac{q_{1}}{p_{1}} \cdot \frac{q_{2}}{p_{2}} \cdots \frac{q_{N}}{p_{N}}\right)$. The period of the representative of the first subshrub level $m_{1}$ branch $d_{11} d_{12} \cdots d_{1 m_{1}}$ is

$$
p_{r_{1}}=p_{1} p_{2} \cdots p_{N-1}\left(p_{N}+\Sigma_{1}\right)
$$

where $\Sigma_{1}=d_{11}+d_{12}+\cdots+d_{1 m_{1}}$. The period of the representative of the second subshrub level $m_{2}$ branch $d_{11} d_{12} \cdots d_{1 m_{1}} \overline{d_{1\left(m_{1}+1\right)}} \rightarrow d_{21} d_{22} \cdots d_{2 m_{2}}$ is

$$
p_{r_{2}}=p_{1} p_{2} \cdots p_{N-2}\left[p_{N-1}\left(p_{N}+\Sigma_{1}-1\right)+\Sigma_{2}\right] \text {, }
$$

where $\Sigma_{1}=d_{11}+d_{12}+\cdots+d_{1 m_{1}}$ and $\Sigma_{2}=d_{21}+d_{22}+\cdots+d_{2 m_{2}}$. And so on. The period of the representative of the $N^{\text {th }}$ subshrub level $m_{N}$ branch $d_{11} d_{12} \cdots d_{1 m_{1}} \overline{d_{1\left(m_{1}+1\right)}} \rightarrow d_{21} d_{22} \cdots d_{2 m_{2}} \overline{d_{2\left(m_{2}+1\right)}} \rightarrow$ $\cdots \rightarrow d_{N 1} d_{N 2} \cdots d_{N m_{N}}$ is

$$
\begin{aligned}
& p_{r_{5}}=p_{1}\left[p_{2} \cdots\left[p_{N-1}\left(p_{N}+\Sigma_{1}-1\right)+\Sigma_{2}-1\right] \cdots+\Sigma_{N-1}\right. \\
& -1]+\Sigma_{N},
\end{aligned}
$$

where $\Sigma_{1}=d_{11}+d_{12}+\cdots+d_{1 m_{1}}, \Sigma_{2}=d_{21}+d_{22}+\cdots+d_{2 m_{2}}, \ldots$, and $\Sigma_{N}=d_{N 1}+d_{N 2}+\cdots+d_{N m_{N}}$.

To simplify these equations we extend the expressions of Eq. (40)

$$
\begin{aligned}
& p_{1}^{\prime}=p_{1}\left(p_{2}^{\prime}-1\right)+\Sigma_{N}, \\
& p_{2}^{\prime}=p_{2}\left(p_{3}^{\prime}-1\right)+\Sigma_{N-1}, \\
& \vdots \\
& p_{N}^{\prime}=p_{N}+\Sigma_{1}
\end{aligned}
$$

and the Eqs. (47)-(49) can be simplified to

$$
\begin{aligned}
& p_{r_{1}}=p_{1} p_{2} \cdots p_{N-1} p_{N}^{\prime}, \\
& p_{r_{2}}=p_{1} p_{2} \cdots p_{N-2} p_{N-1}^{\prime}, \\
& p_{r_{N}}=p_{1}^{\prime} .
\end{aligned}
$$

If we introduce the index $i$ that is equal to $1,2 \cdots N$ for the first, second $\ldots N^{\text {th }}$ subshrub, we can bring the Eqs. (51) - (53) in only one

$$
p_{r_{i}}=p_{1} \cdots p_{N-i} p_{N+1-i}^{\prime}
$$

where $p_{N-i+1}^{\prime}$ can be computed from Eq. (50) and $p_{1} \cdots p_{N-i}$ becomes 1 when $i=N$. Equation (54) calculates the period of any branch representative of any subshrub $i$ of any $N$-ary shrub of the Mandelbrot set. 


\subsection{Generalization for nodes}

As we saw in section 3.3, the preperiod $n^{*}$ of a node is related to the period $p_{r_{i}}$ of the branch representative (when the associated number of both node and branch are the same) by $n^{*}=p_{r_{i}}+1$. Furthermore, it is easy to generalize Eqs. (23), (27) and (31), that we saw for a tertiary component, to obtaining the period $p^{*}$ of a node. Therefore, a node of the $\operatorname{subshrub}_{i}\left(\frac{q_{1}}{p_{1}} \cdot \frac{q_{2}}{p_{2}} \cdots \frac{q_{N}}{p_{N}}\right), 1 \leq i \leq N$, is the Misiurewicz point $\mathrm{M}_{n^{*}, p^{*}}$ with

$$
n^{*}=p_{1} \cdots p_{N-i} p_{N-i+1}^{\prime}+1, p^{*}=p_{1} \cdots p_{N-i}
$$

where $p_{N-i+1}^{\prime}$ can be computed from Eq. (50) and $p_{1} \cdots p_{N-i}$ becomes 1 when $i=N$. Equation (55) calculates the preperiod and the period of any node of any subshrub $i$ of any $N$-ary shrub of the Mandelbrot set.

\subsection{Generalization for tips}

We begin by the normal case for the $i^{\text {th }}$ shrub, when $\Sigma_{1}=0 \cup d_{i m_{i}}>d_{i\left(m_{i}+1\right)}$. If we generalize for the case of a $N$-ary hyperbolic component of the form $\frac{q_{1}}{p_{1}} \cdot \frac{q_{2}}{p_{2}} \cdots \frac{q_{N}}{p_{N}}$ and if we take into account the expressions (21), (24) and (27) that we saw for the normal case of a tertiary hyperbolic component, then the tips of subshrub $1\left(\frac{q_{1}}{p_{1}} \cdot \frac{q_{2}}{p_{2}} \cdots \frac{q_{N}}{p_{N}}\right)$, subshrub $\left(\frac{q_{1}}{p_{1}} \cdot \frac{q_{2}}{p_{2}} \cdots \frac{q_{N}}{p_{N}}\right), \cdots, \operatorname{subshrub}_{N}\left(\frac{q_{1}}{p_{1}} \cdot \frac{q_{2}}{p_{2}} \cdots \frac{q_{N}}{p_{N}}\right)$ are Misiurewicz points $\mathrm{M}_{n^{*}, p^{*}}$ with

$$
\begin{aligned}
& n^{*}=p_{1} \cdots p_{N-1}\left(p_{N}+\Sigma_{1}-d_{1\left(m_{1}+1\right)}\right)+1, \\
& p^{*}=p_{1} \cdots p_{N-1} d_{1\left(m_{1}+1\right)}, \\
& n^{*}=p_{1} \cdots p_{N-2}\left[p_{N-1}\left(p_{N}+\Sigma_{1}-d_{1\left(m_{1}+1\right)}\right)\right. \\
& \left.+\Sigma_{2}-d_{2\left(m_{2}+1\right)}\right]+1, p^{*}=p_{1} \cdots p_{N-2} d_{2\left(m_{2}+1\right),} \\
& \vdots \\
& n^{*}=p_{1}\left[\cdots \left[p _ { N - 2 } \left[p_{N-1}\left(p_{N}+\Sigma_{1}-d_{1\left(m_{1}+1\right.}\right)\right.\right.\right. \\
& \left.\left.\left.+\Sigma_{2}-d_{2\left(m_{2}+1\right)}\right]+\Sigma_{3}-d_{3\left(m_{3}+1\right)}\right]+\cdots\right] \\
& +\Sigma_{N}-d_{N\left(m_{N}+1\right)}+1, p^{*}=d_{N\left(m_{N}+1\right)},
\end{aligned}
$$

respectively. We have given again the non-simplified form of Eqs. (56) - (58) even though we know that $d_{1\left(m_{1}+1\right)}=d_{2\left(m_{2}+1\right)}=\cdots=d_{(i-1)\left(m_{i-1}+1\right)}=1$ for tip $i$. In order to simplify these expressions, we can define

$$
\begin{aligned}
& p_{1}^{\prime \prime}=p_{1} p_{2}^{\prime \prime}+\Sigma_{N}-d_{N\left(m_{N}+1\right)}, \\
& p_{2}^{\prime \prime}=p_{2} p_{3}^{\prime \prime}+\Sigma_{N-1}-d_{(N-1)\left(m_{N-1}+1\right)}, \\
& \vdots \\
& p_{N}^{\prime \prime}=p_{N}+\Sigma_{1}-d_{1\left(m_{1}+1\right)} .
\end{aligned}
$$

By taking into account these expressions, Eqs. (56) - (58) can be simplified as

$$
\begin{aligned}
& n^{*}=p_{1} \cdots p_{N-1} p_{N}^{\prime \prime}+1, \\
& p^{*}=p_{1} \cdots p_{N-1} d_{1\left(m_{1}+1\right)}, \\
& n^{*}=p_{1} \cdots p_{N-2} p_{N-1}^{\prime \prime}+1,
\end{aligned}
$$




$$
\begin{aligned}
& p^{*}=p_{1} \cdots p_{N-2} d_{2\left(m_{2}+1\right)}, \\
& \vdots \\
& n^{*}=p_{1}^{\prime \prime}+1, p^{*}=d_{N\left(m_{N}+1\right)} .
\end{aligned}
$$

If we introduce the index $i$ that is equal to $1,2 \ldots N$ for the first, second $\ldots N^{\text {th }}$ subshrub, we can bring the Eqs. (60)-(62) in only one

$$
\begin{aligned}
& n^{*}=p_{1} p_{2} \cdots p_{N-i} p_{N-i+1}^{\prime \prime}+1, \\
& p^{*}=p_{1} p_{2} \cdots p_{N-i} d_{i\left(m_{i}+1\right)},
\end{aligned}
$$

where $p_{N-i+1}^{\prime \prime}$ can be computed from Eq. $(59), d_{1\left(m_{1}+1\right)}=\cdots=d_{(i-1)\left(m_{i-1}+1\right)}=1$ and $p_{1} \cdots p_{N-i}$ becomes 1 when $i=N$. Equation (64) calculates the preperiod and the period of any tip of any subshrub $i$ of any $N$-ary shrub of the Mandelbrot set.

Let us consider now the especial case. If we are in the especial case $\left(\Sigma_{i} \neq 0 \cap d_{i m}<d_{i(m+1)}\right)$, to calculate a tip of the $\operatorname{subshrub}_{i}\left(\frac{q_{1}}{p_{1}} \cdot \frac{q_{2}}{p_{2}} \cdots \frac{q_{N}}{p_{N}}\right)$ we can use the Eq. (63) but now we have to put $\Sigma_{i}-d_{i m_{i}}$ instead of $\Sigma_{i}-d_{i\left(m_{i}+1\right)}$.

\subsection{Generalization for ftips}

If we follow the p.u.p. $1,11, \cdots, \overline{1}$ in the first, second $\ldots$ and $N^{\text {th }}$ subshrub of the shrub $\left(\frac{q_{1}}{p_{1}} \cdot \frac{q_{2}}{p_{2}} \cdots \frac{q_{N}}{p_{N}}\right)$ we reach the more prominent tip, that we shall call it the ftip of the shrub as we saw in former cases. Now $\Sigma_{1}=\Sigma_{2}=\cdots=\Sigma_{N}=0$ and $d_{1\left(m_{1}+1\right)}=d_{2\left(m_{2}+1\right)}=\cdots=d_{N\left(m_{N}+1\right)}=1$. Therefore, Eq. (63) becomes a much more simplified one, and the ftip $\left(\frac{q_{1}}{p_{1}} \cdot \frac{q_{2}}{p_{2}} \cdots \frac{q_{N}}{p_{N}}\right)$ of a $N$-ary hyperbolic component is a Misiurewicz point $\mathrm{M}_{n^{*} p^{*}}$ with

$$
n^{*}=p_{1}^{\prime \prime}+1, p^{*}=1,
$$

where $p_{1}^{\prime \prime}$ can be computed from Eq. (59).

\section{Epilogue}

We have fulfilled our goal of ordering of the most important hyperbolic components (structural ones) and Misiurewicz points of the Mandelbrot set, and we can calculate the period of the former ones and the preperiod and period of the later ones. We have only tackled one hyperbolic component per structural branch, and in fact there is an infinity of them. However, this is a necessary first step in the ordering of the Mandelbrot set hyperbolic components.

\section{Acknowledgements}

This work was supported by Ministerio de Ciencia y Tecnología of Spain, research grant TIC2001-0586.

\section{References}

Beck, C. [1999] "Physical meaning for Mandelbrot and Julia sets," Physica D 125, 171-182.

Branner, B. [1989] "The Mandelbrot set," in Chaos and Fractals: the mathematics behind the computer graphics, eds.

Devaney, R. L. \& Keen L. (AMS, Providence) pp. 75-105.

Crutchfield, J.P. \& Kaneco, K. [1987] "Phenomenology of spatio-temporal chaos," in Directions in chaos Vol. I, ed. Hao, B. L. (World Scientific, Singapore) pp. 272-353. 
Devaney, R.L. [1995] "The fractal geometry of the Mandelbrot set. How to count and how to add," Fractals 3(4), 629-640.

Devaney, R.L. "The Mandelbrot set and the Farey tree," available from http://math.bu.edu /people/bob/papers.html.

Douady, A. \& Hubbard, J. H. [1985] "Etude dynamique des polynômes complexes," Publ. Math. d'Orsay, 85-04. pp. 56-60.

Douady, A. [1986] "Algorithms for computing angles in the Mandelbrot set," in Chaotic Dynamics and Fractals, eds. Barnsley, M. \& Demko, S.G. (Academic Press, New York) pp. 155-168.

Gilbert, E.N. \& Riordan, J. [1961] "Symmetry types of periodic sequences," Illinois J. Math. 5, 657-665.

Grebogi, C., Ott, E. \& Yorke, J. A. [1982] "Chaotic attractors in crisis," Phys. Rev. Lett. 48, 1507-1510.

Hao, B.-L. \& Xie, F.-G. [1993] "Chaotic systems: counting the number of periods," Physica A 194, $77-85$.

Keller, K. [2000] Invariant factors, Julia equivalencies, and the (abstract) Mandelbrot set (Lecture Notes in Mathematics 1732, Springer, Berlin).

Lau, E. \& Schleicher, D. [1994] "Internal addresses in the Mandelbrot set and irreducibility of polynomials," IMS Preprint 94-19 available from http://www.\%20math.sunysb.edu/cgi-bin/preprint.pl?ims94-19.

Lorenz, E. N. [1963] "Deterministic nonperiodic flow," J. Atmos. Sci. 20(2), 130-141.

Lutzky, M. [1993] "Counting hyperbolic components of the Mandelbrot set," Phys. Lett. A 177, 338-340.

Mandelbrot, B.B. [1980] "Fractal aspects of the iteration of $z \rightarrow \lambda z(1-z)$ for complex $\lambda$ and $z$," in Nonlinear Dynamics, ed. Helleman, R.H.G. (Annals of the New York Academy of Sciences, New York) pp. 249-259.

Mandelbrot, B.B. [1983] "On the quadratic mapping $z \rightarrow z^{2}-\mu$ for complex $\mu$ and $z$ : the fractal structure of its M set, and scaling," Physica D 7, 224-239.

May, R. [1976] "Simple mathematical models with very complicated dynamics," Nature 261, 459-467.

Metropolis, N., Stein, M. L. \& Stein P. R. [1973] "On finite limit sets for transformations on the unit interval," J. Comb. Theory 15(1), 25-44.

Milnor, J. [1989] "Self-similarity and hairiness in the Mandelbrot set," in Computers in Geometry and Topology, ed. Tangora (Lecture Notes in Pure and Applied Mathematics 114, Dekker) pp. 211-257.

Milnor, J. [2000] "Periodic orbits, external rays and the Mandelbrot set: an expository account," Asterisque 261, 277-333. Available from http://www.\%20math.sunysb.edu/cgi-bin/preprint.pl?ims 99-3.

Misiurewicz, M. \& Nitecki Z. [1991] "Combinatorial patterns for maps of the interval," Mem. Am. Math. Soc. 94(456), 1-110.

Myrberg, P.J. [1963] "Iteration der reellen polynome zweiten grades III," Ann. Acad. Sci. Fenn.-M. 336 (3), 1-18.

Munafo, R. [1999] "R2 naming system" available from http://www.geocities.com/gigi_avatar27 /pub/muency/r2namingsystem.html.

Pastor, G., Romera, M. \& Montoya, F. [1996a] "An approach to the ordering of one-dimensional quadratic maps," Chaos, Solitons and Fractals 7(4), 565-584.

Pastor, G., Romera, M. \& Montoya, F. [1996b] "On the calculation of Misiurewicz patterns in onedimensional quadratic maps," Physica A 232, 536-553.

Pastor, G., Romera, M. \& Montoya, F. [1997] "Harmonic structure of one-dimensional quadratic maps," Physical Review E 56(2), 1476-1483.

Pastor, G., Romera, M., Sanz-Martín, J. C. \& Montoya, F. [1998] "Symbolic sequences of onedimensional quadratic map points," Physica A 256, 369-382.

Pastor, G., Romera, M., Álvarez, G. \& Montoya, F. [2001] "Misiurewicz point patterns generation in one-dimensional quadratic maps," Physica A 292, 207-230.

Peitgen, H.O. \& Richter, P. H. [1986] The Beauty of Fractals (Springer-Verlag, Berlin Heidelberg).

Romera, M., Pastor, G. \& Montoya F. [1996] "Misiurewicz points in one-dimensional quadratic maps," Physica A 232, 517-535. 
Romera, M., Pastor, G., Alvarez G. \& Montoya F. [1998] "Heredity in one-dimensional quadratic maps," Physical Review E 58(6), 7214-7218.

Ruelle, D. \& Takens, F. [1971] "On the nature of turbulence," Commun. Math. Phys. 20, 167-192.

Sharkovsky, A.N. [1964] "Coexistence of cycles of a continuous transformation of a line into itself," Ukrain. Mat. Zhurn. 16(1), 61-71.

Sharkovsky, A.N., Maistrenko, Yu.L. \& Romanenko, E. Yu. [1993] Difference Equations and their Applications (Kluwer Academic Publishers, Dordrecht).

Stephenson, J. [1992] "Formulae for cycles in the Mandelbrot set III," Physica A 190, 117-129.

Stephenson, J. [1994] "Spirals in the Mandelbrot set III," Physica A 205, 656-664.

Xie, F.-G \& Hao B.-L [1994] "Counting the number of periods in one-dimensional maps with multiple critical points," Physica A 202, 237-263. 